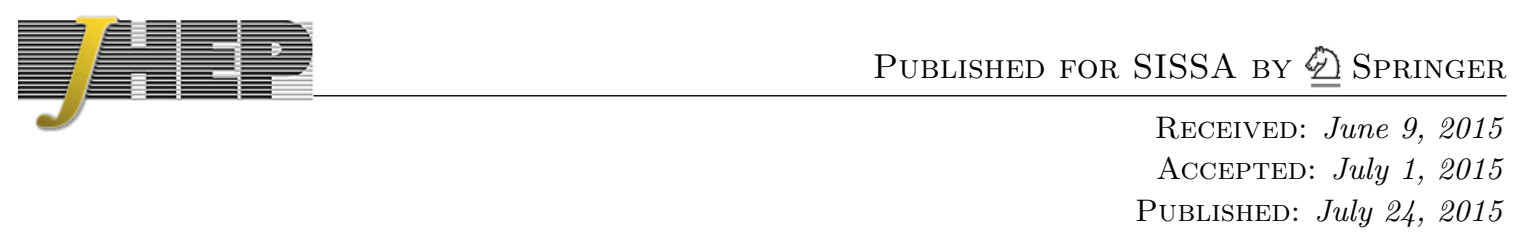

\title{
Signatures of large composite Dark Matter states
}

\author{
Edward Hardy, ${ }^{a, b}$ Robert Lasenby, ${ }^{a}$ John March-Russell ${ }^{a, c}$ and Stephen M. West ${ }^{d}$ \\ ${ }^{a}$ Rudolf Peierls Centre for Theoretical Physics, University of Oxford, \\ 1 Keble Road, Oxford, OX1 3NP, U.K. \\ ${ }^{b}$ Abdus Salam International Centre for Theoretical Physics, \\ Strada Costiera 11, 34151, Trieste, Italy \\ ${ }^{c}$ Stanford Institute for Theoretical Physics, Department of Physics, Stanford University, \\ 382 Via Pueblo Mall, Stanford, CA 94305, U.S.A. \\ ${ }^{d}$ Physics Department, Royal Holloway, University of London, \\ Egham Hill, Egham, Surrey, TW20 0EX, U.K. \\ E-mail: ehardy@ictp.it, robert.lasenby@physics.ox.ac.uk, \\ jmr@thphys.ox.ac.uk, stephen.west@rhul.ac.uk
}

ABSTRACT: We investigate the interactions of large composite dark matter (DM) states with the Standard Model (SM) sector. Elastic scattering with SM nuclei can be coherently enhanced by factors as large as $A^{2}$, where $A$ is the number of constituents in the composite state (there exist models in which $\mathrm{DM}$ states of very large $A \gtrsim 10^{8}$ may be realised). This enhancement, for a given direct detection event rate, weakens the expected signals at colliders by up to $1 / A$. Moreover, the spatially extended nature of the DM states leads to an additional, characteristic, form factor modifying the momentum dependence of scattering processes, altering the recoil energy spectra in direct detection experiments. In particular, energy recoil spectra with peaks and troughs are possible, and such features could be confirmed with only $\mathcal{O}(50)$ events, independently of the assumed halo velocity distribution. Large composite states also generically give rise to low-energy collective excitations potentially relevant to direct detection and indirect detection phenomenology. We compute the form factor for a generic class of such excitations - quantised surface modes - finding that they can lead to coherently-enhanced, but generally sub-dominant, inelastic scattering in direct detection experiments. Finally, we study the modifications to capture rates in astrophysical objects that follow from the elastic form factor, as well as the effects of inelastic interactions between DM states once captured. We argue that inelastic interactions may lead to the DM collapsing to a dense configuration at the centre of the object.

Keywords: Beyond Standard Model, Cosmology of Theories beyond the SM

ArXiv EPrint: 1504.05419 


\section{Contents}

1 Introduction 1

2 Modifications to elastic scattering and direct detection 3

2.1 Dark sector form factors 3

2.2 Direct detection recap 5

2.3 Coherent enhancement of scattering rates 6

$\begin{array}{lll}2.4 & \text { Recoil spectrum from size distribution } & 7\end{array}$

2.5 Dependence on DM velocity distribution 8

2.6 Detectability of a rising energy recoil spectrum 9

3 Low-energy excitations \& inelastic scattering $\quad 10$

$\begin{array}{lll}3.1 \text { Properties of low-energy excitations } & 10\end{array}$

$\begin{array}{lll}3.2 & \text { Inelastic scattering form factors } & 13\end{array}$

$\begin{array}{lll}3.3 & \text { Inelastic recoil spectra } & 13\end{array}$

4 Astrophysical capture $\quad 14$

$\begin{array}{lll}4.1 & \text { Effect of dark form factors on capture rate } & 16\end{array}$

$\begin{array}{lll}4.2 & \text { Self-interactions of captured dark matter } & 18\end{array}$

$\begin{array}{lll}\text { 4.2.1 Dissipative collisions } & 18\end{array}$

$\begin{array}{lll}4.2 .2 & \text { Fusions } & 20\end{array}$

5 Conclusions $\quad 20$

$\begin{array}{ll}\text { A Matrix elements for inelastic scattering } & 21\end{array}$

B High-momentum scattering from large composite states $\quad 23$

$\begin{array}{ll}\text { B.1 Coherent scattering } & 23\end{array}$

B.2 Incoherent scattering 24

$\begin{array}{ll}\text { C Statistical identification of rising distributions } & 24\end{array}$

$\begin{array}{ll}\text { D Solar capture of heavy WIMPS } & 25\end{array}$

\section{Introduction}

Most models of dark matter (DM) assume that it can, for practical purposes, be treated as a collection of point-like particles. However, this is not necessarily the case, and a variety of models in which DM is a composite state have been proposed (for example, WIMPonium [1-3], and dark atoms [4-7]). Given the centrality of DM to our present thoughts about beyond-the-Standard-Model physics, and our present lack of knowledge concerning many of its fundamental characteristics, and thus how DM may reveal itself in 
experiments or observations, it is important to consider possible variations away from the standard picture. This is especially the case if there are qualitatively new features compared to traditional DM models that may affect direct and/or indirect detection phenomenology.

In this paper, we consider the consequences for present-day scattering processes of a simple kind of compositeness, in which the DM states are composed of a large number, $A$, of constituents, forming an extended semi-uniform object, analogous to Standard Model (SM) nuclei formed out of constituent nucleons. ${ }^{1}$ Models that realise such a scenario include Q-balls (non-topological solitons carrying a conserved charge) [9, 10], and Nuclear Dark Matter models, in which DM is made up of bound states of strongly-interacting constituents with short-range interactions [11-13]. The early-universe cosmology of these models has interesting features, as investigated in [10-13]; here, we simply assume that a late-time population of such states exists.

This large and extended compositeness affects elastic scattering and the associated direct detection phenomenology, as we discuss in section 2. Large composite states containing $A$ constituents can have low-momentum-transfer elastic scattering cross sections coherently enhanced by a factor as large as $A^{2}[11,13,14]$. This case is realised if the size of the state is not too large compared to the inverse momentum exchange relevant in direct detection scatterings. In this situation, and assuming that the mass of the composite state is $\propto A$, so the $\mathrm{DM}$ number density is $\propto 1 / A$, the event rate at direct detection experiments will effectively be enhanced $\propto A$ for a given interaction strength between SM and DM constituents. Previous studies of the build-up of composite DM states in the early universe show that large values of $A\left(\gtrsim 10^{8}\right)$ could plausibly be realised [13], so the effective enhancement can be significant.

In addition, if a high enough proportion of the DM states have radii larger than SM nuclei, but not so large as to significantly suppress coherent scattering, the spatial extension of the DM states leads to a dark form factor modifying the momentum dependence of DM-SM scattering (as previously considered in [14]). In the simplest case of a scalar interaction depending only on the density, this form factor has a characteristic series of peaks and troughs, analogous to SM nuclear form factors. In direct detection experiments with sufficiently good energy resolution, these could lead to the striking signature of rises in the energy recoil spectrum. We find that significant features of this kind could be distinguished from point-like elastic scattering after the observation of only $\mathcal{O}(50)$ events, independently of assumptions about the DM halo velocity distribution. A distribution over DM sizes may average out these peaks into a smoothly-falling effective form factor, in many cases resembling that from e.g. the exchange of an intermediate-mass mediator, but multiple direct detection experiments using different SM nuclear targets would still be able to separate out the momentum dependence from halo velocity distribution effects, as with other models of dark form factors [14-18].

As we discuss in section 3, large composite states also generically give rise to longwavelength and low-energy collective excitations. There will also be higher-energy excitations, and fission processes, but making the binding energy scale low enough for these to be

\footnotetext{
${ }^{1}$ The generic scattering phenomenology of small-number composite DM states has been considered in [8].
} 
relevant in direct detection experiments generally complicates the cosmology of such models [13]. We focus on the most model-independent possibility of collective density excitations. We find that they can be of sufficiently low energy to be excited in collisions with SM nuclei, leading to coherently-enhanced inelastic scattering. Although this is sub-dominant to elastic scattering in the scenarios we consider, a non-negligible fraction of events may be inelastic, and we calculate the associated form factor in leading approximation. ${ }^{2}$

As is well known, couplings to SM nuclei may also lead to the capture of DM by astrophysical objects. As discussed in section 4 we find that the composite nature of the DM changes the overall capture rate. In addition, perhaps the most interesting qualitative effect is that the large local DM density inside the star, and the natural possibility of inelastic DM self-interactions, could lead to a DM distribution that is significantly modified compared to the naive expectation. In particular, for either dissipative collisions or fusions, there is the possibility that this process runs away to a state in which most of the captured DM lies in a single very dense configuration. ${ }^{3}$ There may be model-dependent consequences of the energy released by inelastic DM self-interactions, which in the case of fusions could release large amounts of energy into small volumes over short timescales.

\section{Modifications to elastic scattering and direct detection}

\subsection{Dark sector form factors}

For elastic scattering between a point-like state and a spatially extended state, interacting via short-range interactions sourced by some density $\rho$ on the extended side, the dependence on the spatial properties is summarised by the form factor

$$
F(\mathbf{q})=\int d \mathbf{r} e^{i \mathbf{q} \cdot \mathbf{r}} \rho(\mathbf{r}),
$$

i.e. by the Fourier transform of the density. For scattering of DM states off SM nuclei, effective field theory arguments show that the scattering operator on the SM side should take on one of a restricted number of forms (see e.g. [19] for a recent comprehensive analysis). The dark sector density must have a complementary tensorial structure, but could in general be determined by any properties of the state, e.g. density, spin, etc.

In the case of two spatially extended states scattering off each other, the effective density is the convolution of the separate spatial profiles, so the matrix element is found by multiplying the form factors together. Thus, the overall form factor for DM-SM scattering will be $F_{N}(\mathbf{q}) F_{X}(\mathbf{q})$, where $F_{N}$ and $F_{X}$ are, respectively, the SM nuclear form factor and the dark sector form factor (as we will discuss below, in many experimental circumstances the

\footnotetext{
${ }^{2}$ Taking the possibility of inelastic DM self-interactions further, we may also expect, in the context of galactic halos, exothermic DM-DM interactions, e.g, from fusions. This can lead to an increase in the average kinetic energy of DM in the galactic halo, which might in turn result in a clearing out of the central high-density region. This possibility was outlined in [13], and requires a dedicated study of halo dynamics. In this work, we focus on the interactions of composite DM states with the SM, and leave the topic of modified halo dynamics to a future paper.

${ }^{3}$ Since the large composite states with saturated densities we consider in this paper require a short-range repulsive interaction between constituents, forming a black hole at the centre of the star would generally require accumulating a very large DM mass.
} 


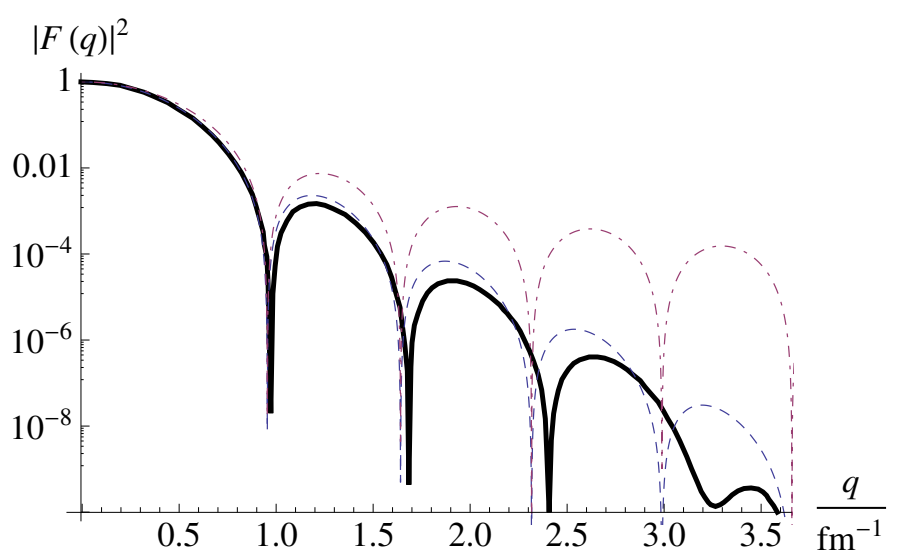

Figure 1. Form factor for nuclear charge density (black) of ${ }^{70} \mathrm{Ge}$ nucleus, as derived from electron scattering data [20]. Blue (dashed) curve is standard Helm parameterisation, red (dot-dashed) curve is constant-density approximation.

SM nuclear form factor is of limited importance). The specific density determining the form factor will depend on the nature of the DM-SM interaction. For simplicity, in this paper we will restrict ourselves to considering the case of a scalar form factor depending only on the number density of the state, e.g. arising from the exchange of a heavy scalar mediator coupling uniformly to all of the constituents. Taking, as discussed in the Introduction, the the composite DM states to consist of an approximately uniform density of constituent matter, a first approximation for the density is a spherical top hat function, leading to a spherical Bessel function form factor,

$$
F(q)=\frac{3 A j_{1}(q R)}{q R}=\frac{3 A(\sin (q R)-q R \cos (q R))}{(q R)^{3}},
$$

where $R$ is the radius of the top hat density, and $A$ is the total volume integral of the source across the distribution, so $F(0)=A$.

SM nuclei provide an example of this kind of roughly-constant-density state, and illustrate the kind of deviations from the top-hat form factor that might occur. Figure 1 shows an example of the form factor corresponding to the nuclear charge density for the particular isotope ${ }^{70} \mathrm{Ge}[20]$, as inferred from electron scattering data. Generally, and in this specific case, the first few peaks and troughs of SM nuclear form factors are well-approximated by the 'Helm' functional form

$$
F(q)=\frac{3 A j_{1}(q R)}{q R} e^{-q^{2} s^{2} / 2},
$$

which is simply the top hat form factor modified to have a finite-width fall-off, over the 'skin depth' $s \simeq 0.9 \mathrm{fm}$ (comparable to the scale of the individual nucleons).

Importantly, in the case of interest to us where both a dark-sector and SM form-factor are present, if the dark states have larger radii than SM nuclei, then the first zeros, or more generally troughs, of $F_{X}(q)$ will occur at smaller $q$ than for $F_{N}(q)$. This means that, while the SM nuclear form factor is of limited importance in most direct detection experiments, the dark form factor may have interesting structure in precisely the momentum, and thus recoil energy, ranges being probed. 


\subsection{Direct detection recap}

In this section, we briefly review the standard direct detection formalism leading to the differential event rate (equation (2.7)), mainly to establish notation for what follows. Readers familiar with this material may safely proceed to section 2.3 .

The differential scattering rate (event rate per unit target mass) at which incident DM particles scatter off an initially-stationary target SM nucleus, giving it recoil energy $E_{R}$, is given by

$$
\frac{d R}{d E_{R}}=\left.\frac{1}{m_{N}} \int_{v>v_{\min }} d^{3} \mathbf{v} n_{X} f(\mathbf{v}) v \frac{d \sigma_{X N}}{d E_{R}}\right|_{v},
$$

where $v \equiv|\mathbf{v}|, m_{N}$ is the mass of the SM nucleus, $n_{X} f(\mathbf{v}) d^{3} \mathbf{v}$ is the differential number density of incident $\mathrm{DM}$ particles, $v_{\min }$ is the minimum velocity required to obtain recoil energy $E_{R}$, and $\sigma_{X N}$ is the DM-nucleus scattering cross section. ${ }^{4}$ For non-relativistic elastic scattering, $v_{\min }=\sqrt{\frac{E_{R} m_{N}}{2 \mu_{X N}^{2}}}$, where $\mu_{X N}=\frac{m_{X} m_{N}}{m_{X}+m_{N}}$ is the DM-nucleus reduced mass.

The momentum transfer in an elastic collision is $q=\mu_{X N} v \sqrt{2\left(1-\cos \theta^{*}\right)}$, where $\theta^{*}$ is the scattering angle the CoM frame. Since $E_{R}=\frac{q^{2}}{2 m_{N}}$, we have $d E_{R}=\frac{2 \mu_{X N}^{2} v^{2}}{m_{N}} \frac{d \Omega^{*}}{4 \pi}$, where $d \Omega^{*}$ is differential solid angle in the CoM frame. Referring back to the discussion of section 2.1, the matrix element for scattering at angle $\theta^{*}$ will depend on the momentum transfer $\mathbf{q}$, and possibly also on the velocity $\mathbf{v}$. Treating isotropic, velocity-independent scattering to start with, and writing $\frac{1}{4 \pi} \sigma_{X N}(q) \equiv \frac{d \sigma}{d \Omega^{*}}$, we have

$$
\left.\frac{d \sigma_{X N}}{d E_{R}}\right|_{v}=\frac{m_{N}}{2 \mu_{X N}^{2} v^{2}} \sigma_{X N}(q)
$$

So, under the assumption of velocity-independent scattering, we can factor the differential scattering rate as

$$
\frac{d R}{d E_{R}}=\left(\int_{v>v_{\min }} d^{3} \mathbf{v} \frac{f(\mathbf{v})}{v}\right) \frac{n_{X}}{2 \mu_{X N}^{2}} \sigma_{X N}(q) \equiv g\left(v_{\min }\right) \frac{n_{X}}{2 \mu_{X N}^{2}} \sigma_{X N}(q) .
$$

Altering notation somewhat from section 2.1, we can write $\sigma_{X N}(q)=\sigma_{X N} F_{N}(q)^{2} F_{X}(q)^{2}$, where $\sigma_{X N}$ is the zero-momentum-transfer cross section (including any coherence enhancement), so that $\left|F_{N}(0)\right|=\left|F_{X}(0)\right|=1$.

The scattering rate is usually expressed in terms of the DM scattering cross section with nucleons, instead of with full nuclei. For dimension-6 interactions between the DM constituents and SM quarks, the zero-momentum-transfer scattering cross section goes as $|C|^{2} \frac{\mu^{2}}{\Lambda^{4}}$, where $\Lambda$ is the suppression scale associated with the interaction, and $C$ is the coherence enhancement factor. Here, this is given by the product of the integrated densities relevant to the microscopic interaction for the SM nucleus and the DM state, which we take to be simply the respective nucleon numbers. Thus $\sigma_{X N}=\frac{\left|C_{N}\right|^{2}}{\left|C_{n}\right|^{2}} \frac{\mu_{X N}^{2}}{\mu_{X n}^{2}} \sigma_{X n}$, where $\mu_{X n}$

\footnotetext{
${ }^{4}$ Throughout we will consider parameter ranges where the DM-SM interactions are sufficiently weak that the Earth is optically thin to DM, so that $n_{X} f(\mathbf{v})$ is the DM halo distribution at the location of the Earth.
} 

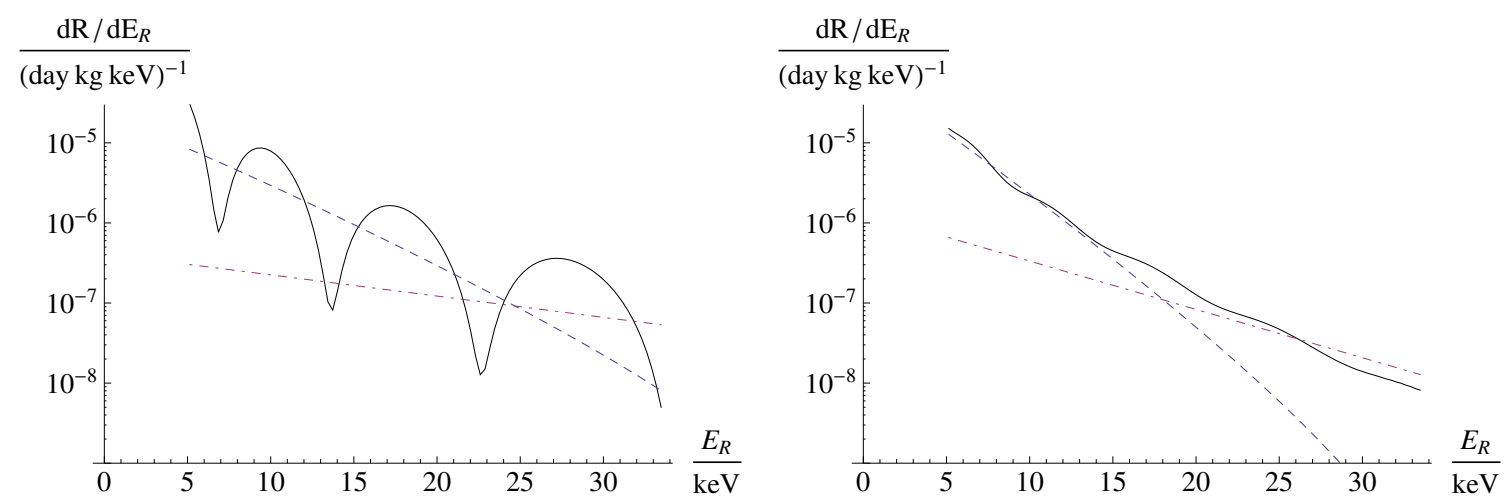

Figure 2. Recoil energy spectrum (black, solid curves) for Bessel-function form factor, radius $50 \mathrm{fm}$, with DM velocity distribution as given by Standard Halo Model [21], assuming Left: Germanium detector target, with Gaussian energy response $\left(\sigma(E)=\sqrt{0.3^{2}+0.06^{2} E / \mathrm{keV}} \mathrm{keV}\right)$, and Right: Xenon detector target, with Gaussian energy response $(\sigma(E)=0.6 \mathrm{keV} \sqrt{E / \mathrm{keV}})$, assuming $5 \mathrm{keV}$ energy threshold for both. The DM state is taken to be composed of $3 \times 10^{6}$ constituents, each of mass $20 \mathrm{GeV}$, with constituent-SM nucleon cross section of $2 \times 10^{-13} \mathrm{pb}$. For comparison, blue (dashed) curves show energy recoil spectrum for a $20 \mathrm{GeV}$ WIMP, red (dot-dashed) for a $1 \mathrm{TeV}$ WIMP, both with $\sigma_{X n}=10^{-9} \mathrm{pb}$. Energy response functions are taken from projections for future experiments in [18].

is the DM-nucleon reduced mass, and so $^{5}$

$$
\frac{d R}{d E_{R}}=g\left(v_{\min }\right) \frac{n_{X}}{2 \mu_{X n}^{2}} \frac{\left|C_{N}\right|^{2}}{\left|C_{n}\right|^{2}} \sigma_{X n} F_{N}(q)^{2} F_{X}(q)^{2} .
$$

\subsection{Coherent enhancement of scattering rates}

With the assumptions made in the previous section, the zero-momentum transfer cross section between a DM state with $A$ constituents and a SM nucleus with $N$ constituents is $\sigma_{0} \sim A^{2} N^{2} \frac{\mu_{X N}^{2}}{\Lambda^{4}}$. Taking the mass of the DM state to be $\propto A$, the DM number density is $\propto \frac{1}{m_{\mathrm{X}}} \propto \frac{1}{A}$, assuming temporarily that all of the DM is of the same size. So, the overall scattering rate in direct detection experiments will, for $m_{\mathrm{X}} \gg m_{N}$, be $\propto A$ for fixed SM-constituent interactions. This is true if the DM radius $R$ is small enough that typical momentum transfers do not probe $q R \gtrsim 1$ - otherwise, the scattering rate is suppressed by the DM form factor, as discussed in section 2.1. While, as discussed in section 2.4, there might be some number distribution over DM states with different radii, if the mass distribution and scattering-rate distributions are confined to a small range in logarithmic size, then the $\propto A$ enhancement will hold approximately.

Figure 2 shows an example of the direct detection recoil energy spectra resulting from a scenario of this kind, corresponding to DM particles with a Bessel-function form factor of radius $50 \mathrm{fm}$. These are compared to the recoil spectra for standard momentum-independent (i.e. $F_{X}=1$ ) WIMP scattering. Note that the constituent-SM nucleon cross section required in the composite model is much smaller than that required in WIMP models giving

\footnotetext{
${ }^{5}$ This equation describes what a detector with perfect energy resolution would see. If a detector has some response function $\kappa$ such that the differential rate to detect events with true energy $E_{R}$, occurring at rate $R$, at measured energy $E_{R}^{\prime}$, is $R \kappa\left(E_{R}, E_{R}^{\prime}\right) d E_{R}^{\prime}$, then the differential rate for measured events is $R_{d}\left(E_{R}\right)=\int d E_{R}^{\prime} \kappa\left(E_{R}, E_{R}^{\prime}\right) \frac{d R}{d E_{R}^{\prime}}$.
} 
approximately the same event rates. An additional point is that the much better energy resolution expected in solid-state experiments would enable them to resolve the peaks and troughs of a dark form factor corresponding to much larger radii than for liquid-phase (e.g. Xenon) experiments.

The possible $\propto A$ enhancement means that, for a given direct detection event rate, the expected production of lighter single constituents in SM processes in colliders is reduced. For the example of DM coupling through the Higgs portal, the strongest collider constraint for DM states with $m_{X}<m_{h} / 2$ comes from the Higgs invisible width. As described in [22], a bound of $<10 \%$ on the invisible branching ratio of the Higgs puts constraints on the DM-Higgs coupling a factor of a few better than current direct detection experiments for DM masses a small factor lower than $m_{h} / 2$. Thus, only a modest relative suppression of collider rates vs direct detection rates is needed to render the direct detection bounds more constraining. Taking the example in figure 2, with DM constituents of mass $20 \mathrm{GeV}$ each having a nucleon scattering cross section $\sigma_{n}=2 \times 10^{-13} \mathrm{pb}$, we would require Higgs invisible width bounds of $\sim 10^{-5}$ to be competitive with current direct detection experiments.

\subsection{Recoil spectrum from size distribution}

The previous sections considered a dark form factor arising from DM particles having a single, common size. However, in many models of large composite states (e.g. [13], Q-balls), the cosmological process through which a population of these states arises generates a distribution over multiple sizes, which would lead to a smeared-out signature in energy recoil spectra.

For DM consisting of a set of states $X_{i}$, the recoil spectrum for elastic scattering will be

$$
\frac{d R}{d E_{R}}=F_{N}(q)^{2} \sum_{i} g_{i}\left(v_{\min }\left(\mu_{i, N}\right)\right) \frac{n_{i}}{2 \mu_{i, n}^{2}} \frac{\left|C_{i}\right|^{2}}{\left|C_{n}\right|^{2}} \sigma_{i, n} F_{X, i}(q)^{2},
$$

where the subscripted quantities are those of equation (2.7) for each species $X_{i}$. In the specific case of a spectrum of related states, this may simplify somewhat. Absent astrophysical self-interactions (see section 4.2), all of the states may be expected to have the same velocity distribution, i.e. $g_{i}=g$. From $\sigma_{n} \sim|C|^{2} \frac{\mu_{X n}^{2}}{\Lambda^{4}}$, we expect the $\mu_{i, n}$ dependence to cancel, and additionally, if all of the states are heavy $\left(m_{i} \gg m_{N}\right)$, then $\mu_{i, N} \simeq m_{N}$, so we can factor out the $g$ dependent term fully. Overall,

$$
\begin{aligned}
\frac{d R}{d E_{R}} & \simeq \frac{n_{X}}{2 m_{n}^{2}} F_{N}(q)^{2} g\left(v_{\min }\right) \frac{|C|^{2}}{\left|C_{n}\right|^{2}} \sigma_{n} \sum_{i} \frac{n_{i}}{n_{X}} \frac{\left|C_{i}\right|^{2}}{|C|^{2}} F_{X, i}(q)^{2} \\
& \equiv \frac{n_{X}}{2 m_{n}^{2}} F_{N}(q)^{2} g\left(v_{\min }\right) \frac{|C|^{2}}{\left|C_{n}\right|^{2}} \sigma_{n} F_{X}(q)^{2},
\end{aligned}
$$

where $n_{X} \equiv \sum_{i} n_{i}$, and we have replaced the sum over individual states with a single effective form factor, choosing $C$ appropriately so that $F_{X}(0)=1$.

Figure 3 shows the effect of a distribution over DM states of the kind considered in [13]. The sum over different sizes corresponds to adding together differently scaled form factors, which smooths out the troughs of the Bessel-function form factor. In this case, we end up with a form factor which is similar to that obtained from the exchange of a light mediator particle, $F(q) \propto \frac{1}{q^{2}+m_{\phi}^{2}}$, but arising entirely from contact interactions, without the need 

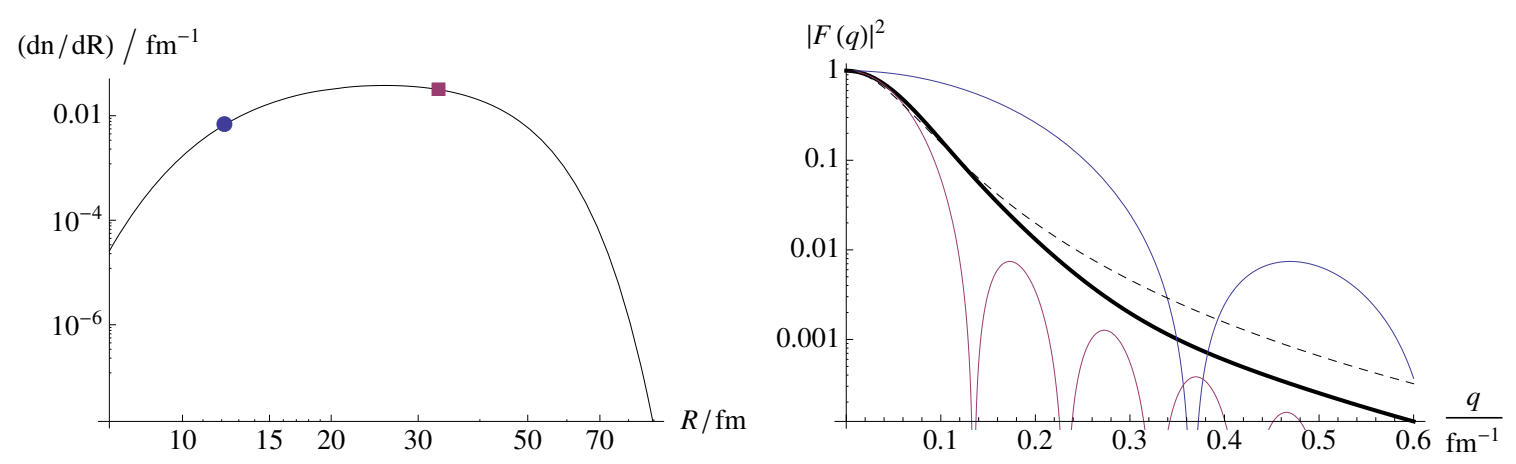

Figure 3. Left: example (non-dimensionalised) number distribution of DM states arising from nucleosynthesis-type process (from [13]). Right: form factors for DM sizes corresponding to points on left-hand plots (upper curve to blue circle, lower curve to red square), and (black) effective form factor for whole number distribution. Dashed line shows form factor for light mediator particle, $F(q) \propto 1 /\left(q^{2}+m^{2}\right)$, with $m=16 \mathrm{MeV}$.

for a light state. ${ }^{6}$ For a given DM velocity distribution, these kinds of dark form factor gives an energy recoil spectrum with a shape different from that of standard momentumindependent scattering, falling off more slowly at high energies than a low-mass WIMP, and more quickly at low energies than a higher-mass one. In the next section, we discuss how such form factors could be distinguished from momentum-independent scattering.

\subsection{Dependence on DM velocity distribution}

From the definition in equation (2.6) of $g\left(v_{\min }\right)=\int_{v>v_{\min }} d^{3} \mathbf{v} \frac{f(\mathbf{v})}{v}$, it is clear that, by choosing $f(\mathbf{v})$ appropriately, we can make $g$ any non-increasing function of $v_{\min }$ (with the physical constraint that it must fall off very fast past $v_{e}+v_{\mathrm{esc}}$, where $v_{e}$ is the velocity of Earth relative to the Galactic rest frame, and $v_{\text {esc }}$ is the Galactic escape velocity at the position of the Earth). Although the Standard Halo Model, which posits a MaxwellBoltzmann DM velocity distribution, is commonly assumed, it is highly plausible that the DM velocity distribution at Earth differs from this, perhaps significantly. ${ }^{7}$

\footnotetext{
${ }^{6}$ While it is possible that a model with an intermediate-mass mediator would have additional phenomenology distinguishing it from the case of large composite DM, this would not have to be the case. Though a light mediator will give rise to self-interactions between galactic DM particles, for heavy (and thus dilute) DM these will not be frequent enough to have detectable effects on halo shapes. The direct detection event rate is set by the products of the squared couplings for the SM and the dark sector, so by making the dark sector coupling large, we could make the required SM coupling very small. Current direct detection experiments are sensitive enough that any future signals would imply a minimum value of the SM coupling small enough to be significantly below any direct production constraints. Of course, the SM coupling may be above this minimum if the dark sector coupling is also small, so direct production signals are not ruled out. Also, as discussed in [13], direct bounds on the SM couplings of light $(m \ll 100 \mathrm{MeV})$ states generally imply that they cannot lose any initial cosmological energy density they have to the SM sufficiently fast (except possibly to neutrinos), requiring the introduction of additional light hidden sector states that persist to the present day.

${ }^{7}$ Simulations of dark halo formation indicate that non-Maxwell-Boltzmann velocity distributions may generically arise [23], and also suggest the possibility of a co-rotating DM disc [24], along with sub-structure including cold streams [25].
} 
This has the consequence that, for elastic scattering, the energy recoil spectrum can be anything of the form $F_{N}(q)^{2} F_{X}(q)^{2}$ multiplied by a non-increasing distribution, as discussed in [26]. So, even though the energy recoil spectrum for the dark form factor looked different from that for a WIMP assuming the same DM velocity distribution, by changing the velocity distribution we could make a WIMP mimic the (averaged) form factor spectrum, since this is decreasing with $E_{R}$.

However, if we have data from multiple experiments, each of which uses a different type of SM target nucleus, this degeneracy can be lifted. On the assumption that there is no dark form factor, the event distributions (once we have compensated for the different target nuclei) with respect to $v_{\min }$ should be the same for each of the experiments [26-31] - any disagreement indicates the presence of some extra effect. For a dark form factor, since $q\left(v_{\min }\right)=2 \mu_{X N} v_{\min }$, changing $\mu_{X N}$ by changing $m_{N}$ will change the range of $F_{X}(q)$ that we sample (significantly so if the DM mass is larger than those of the SM target nuclei). Ref. [18] performs a multi-target analysis along these lines for various DM models, and a forthcoming paper [32] applies this method to the case of heavy DM coupling via an intermediate-mass mediator, which as noted above gives form factors that are very similar to those which may be obtained via averaging over Bessel-function form factors. It is found $^{8}$ that such a form factor can be distinguished from contact-like scattering with only a handful of events in multiple detectors, while effective exposures of only around 1.0 ton years in multiple detectors could, assuming DM-SM cross sections close to current upper bounds, allow the determination of the mediator mass (which, in the composite case, would correspond to the average size of the DM) to around $25 \%$ accuracy.

\subsection{Detectability of a rising energy recoil spectrum}

While sections 2.4 and 2.5 have considered the situation of a distribution over DM sizes, the more striking scenario of DM states being concentrated around a single size, giving rise to peaks and troughs in the energy recoil spectrum as per figure 2 , has qualitatively different detectability in direct detection experiments. As described in the previous section, the energy recoil spectrum for elastic scattering can be anything of the form $F(q)^{2}$ multiplied by a non-increasing distribution. While this allows non-increasing sections of an energy recoil spectrum to be explained by a combination of form factor and DM velocity distribution, any rises in the spectrum must come from the form factor (or from the presence of inelastic scattering). Though summing over multiple Bessel-function widths will generally smooth out the individual peaks into a falling distribution, if the particle sizes were clustered mostly around a particular value, ${ }^{9}$ then the troughs/peaks of such an effective form factor could give a rising recoil spectrum. Assuming a detector has sufficiently good energy resolution so as not to smooth out the troughs in the underlying recoil spectrum, it will be possible, with sufficiently many events, to rule out point-like elastic scattering. Furthermore, if both the falling and rising parts of a trough were visible, this would be a clear sign of a

\footnotetext{
${ }^{8}$ We thank John Cherry for providing plots of the exposure time needed to distinguish our effective form factor.

${ }^{9}$ For example, due to the binding energy per constituent reaching a maximum value at some size, and decreasing after that (as for iron in the SM), rendering larger states unstable to fission.
} 

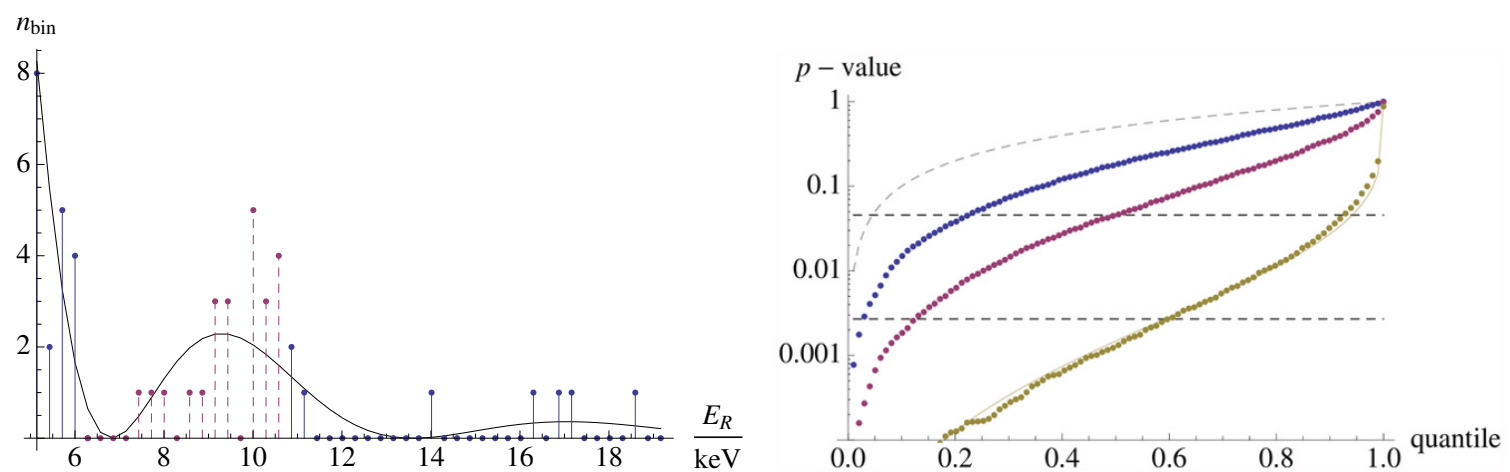

Figure 4. Left: energy recoil spectrum (black) for a Bessel-function form factor corresponding to radius $50 \mathrm{fm}$, with other parameters as per left-hand plot of figure 2. Blue points (solid lines) are a particular sample of 50 events from this distribution, binned with an energy width of $0.3 \mathrm{keV}$. Red points (dashed lines) show the interval of points that is worst fit by a non-increasing distribution (see text). The $p$-value for the test described in appendix $\mathrm{C}$ is 0.005 . Right: cumulative distribution functions (CDFs) for $p$-value from test described in appendix C, for 30 samples (blue), 50 samples (red), and 100 samples (yellow). Grey (dashed) curve shows the CDF for a uniform underlying distribution. The upper and lower dashed lines shows the $p$ values corresponding to $2 \sigma$ and $3 \sigma$ significance for rejecting the hypothesis of a non-increasing distribution.

more complicated momentum-dependent form factor, or of some combination of inelastic scattering modes.

We can test whether a given set of events came from a non-increasing distribution by locating the energy interval with the 'worst' bias towards its high-energy end - here measured simply by the average energy of the events - and asking what the probability is that a given sample from a candidate non-increasing distribution would have an interval that extreme (explained in more detail in appendix C). Figure 4 displays the results of applying this test to simulated data from a model with a Bessel-function dark form factor, scaled so that a number of the peaks/troughs are visible in the energy recoil spectrum. As illustrated, around 12 expected events in the most significant rising section, corresponding here to $\sim 50$ events overall, are sufficient to obtain a two-sigma exclusion of the nonincreasing hypothesis in the majority of cases. While a realistic analysis would need to take into account the distribution of background events and other issues, this illustrates that relatively few events from only a single detector may suffice to give very interesting physical information about DM properties.

\section{Low-energy excitations \& inelastic scattering}

\subsection{Properties of low-energy excitations}

Another feature generic to large composite states is the presence of low-lying modes, leading to the possibility of inelastic scattering in which these modes are excited. Such modes can be broadly characterised as either of "single-particle" or "collective" type. We are most interested in the collective modes since, as we will discuss, their excitation amplitudes can 
be coherently enhanced compared to the single-particle excitations, and thus can dominate the inelastic scattering rate if they are of low enough energy to be excited.

There are a number of forms such collective excitations could take. The simplest and most generic type of collective excitations are simply vibrational modes of either the bulk or of the surface of the large composite state. Alternatively, if the constituents have spins, moments, or other intrinsic properties, then collective oscillations of these properties, such as spin waves, can occur. In addition, if the ground state is not spherically symmetric, so the composite state is deformed in shape as happens for large SM nuclei, then there will be lowlying rotational modes as well. For definiteness, in this paper we will focus on the inelastic scattering involving the most universal and model-independent of these collective modes, namely, the surface and bulk vibrational modes that are of long wavelength compared to the scale of the individual constituents.

For large composite states consisting of roughly uniform 'nuclear matter', the lowestenergy density waves will generally be surface waves, i.e. volume-preserving oscillations of the 'nuclear surface' [33]. The reason for this is that the energy of the lowest bulk compressional excitations is set by the speed of sound for such waves, $c_{c} \sim \sqrt{\frac{K}{\rho}}$, where $K$ parameterises the compressibility of the nuclear material, and $\rho$ is the density. Taking into account that the lowest possible wavenumber is $k \sim 1 / R$, the energy of the low-lying compressional excitations is given by

$$
\delta E_{c} \sim \sqrt{\frac{K}{\rho}} \frac{1}{R} .
$$

On the other hand, the speed of volume-preserving surface capillary waves is set by $c_{s} \sim$ $\sqrt{\frac{\sigma k}{\rho}}$, where $\sigma$ is the surface tension (this also holds in relativistic hydrodynamics [34]), so the energy of the low-lying surface excitations is given by

$$
\delta E_{s} \sim \sqrt{\frac{\sigma}{\rho}} \frac{1}{R^{3 / 2}} .
$$

Using the facts that $R \sim A^{1 / 3} R_{1}$, where $R_{1}$ is the length scale of a single constituent, and that both the surface tension and bulk compressibility are set by the same underlying interaction strength between the basic constituents so we have $\sigma \sim R_{1} K$, leads to

$$
\frac{\delta E_{s}}{\delta E_{c}} \sim A^{-1 / 6} .
$$

Thus for large enough $A$ the energy of the bulk compressional modes is well separated from the lower-lying surface modes.

We now summarise the dynamics of the surface modes more quantitatively. Classically, for small-amplitude surface waves of a homogeneous, incompressible, sharp-edged fluid droplet, we have $\rho(r, \theta, \phi)$ constant inside $R^{\prime}(\theta, \phi, t)$ and zero outside, with

$$
R^{\prime}(\theta, \phi, t)=R\left[1+\sum_{l \geq 2} \sum_{m=-l}^{l} \alpha_{l m}(t) Y_{l m}(\theta, \phi)\right],
$$


for amplitude coefficients $\alpha_{l m}(t)$. The $l=1$ modes are removed as they correspond to oscillations of the CoM position, while the $l=0$ mode is removed as it is just the monopole compression oscillation. ${ }^{10}$ The total Hamiltonian of the surface excitations is

$$
H=\frac{1}{2} \sum_{\substack{l \geq 2, m=-l, \ldots,+l}}\left(B_{l}\left|\dot{\alpha}_{l m}(t)\right|^{2}+C_{l}\left|\alpha_{l m}(t)\right|^{2}\right),
$$

where $B_{l}=\rho R^{5} / l$ and $C_{l}=(l-1)(l+2) R^{2} \sigma$ are the "mass" and "stiffness" parameters of each mode.

Quantising this system leads to each mode being an independent quantum harmonic oscillator. The mode frequencies, $\omega_{l m}$, of the oscillations are independent of the azimuthal spherical harmonic parameter $m$ and are given by [34]

$$
\omega_{l}=\sqrt{\frac{C_{l}}{B_{l}}}=\left(\frac{l(l+2)(l-1) \sigma}{R^{3} \rho}\right)^{1 / 2} .
$$

The overall excitation energy spectrum $\Delta E_{\left\{n_{l}\right\}}=\sum_{l \geq 2} n_{l} \omega_{l}$ is thus set by the occupation numbers $n_{l}$ of the $(2 l+1)$-fold-degenerate $l$-modes. It will be important for our discussion of the coherent excitation probability of these modes that the simple harmonic oscillatorlike wavefunctions associated to each mode are characterised by a length scale, $R \epsilon_{l}$, where the dimensionless parameter $\epsilon_{l}$ is given by

$$
\epsilon_{l}=\frac{1}{\sqrt{2 B_{l} \omega_{l}}} .
$$

(For an individual mode with occupation number $n_{l} \gg 1$ the corresponding classical amplitude of oscillation simply scales as $\left|\alpha_{l}\right| \sim \epsilon_{l} \sqrt{n_{l}}$.) In terms of the underlying parameters of the composite state

$$
\epsilon_{l} \propto A^{-7 / 12}\left(\frac{1}{M_{1} R_{1}^{2} \beta}\right)^{1 / 4}
$$

where $\beta \sim \sigma R_{1}^{2}$ is the energy associated to surface tension, and $M_{1}$ is the mass of individual constituents. As discussed in [35], the hydrodynamic values of $B_{l}$ and $C_{l}$ can differ by factors of $\mathcal{O}(10)$ from those obtained experimentally for SM nuclei, due to the effects of shell structure etc. However, the hydrodynamic approximation should give a good qualitative guide to the properties of the low-lying modes of large composite states.

Finally, as mentioned above, as well as collective modes there may also be modes corresponding to the excitation of 'single constituents'. If the interior of the state consists of degenerate fermionic matter, then excitations which move a state from just below the Fermi surface, through a small change in momentum $\mathbf{q}$ approximately tangential to the surface, will look like quasi-particles of energy $\mathbf{q}^{2} /\left(2 m_{*}\right)$, where the quasi-particle mass $m_{*}$ will often be of order the constituent mass [36]. Since the smallest allowed momentum change is $q \sim$ $1 / R$, the lowest-energy quasi-particle excitation will have energy $\omega \sim \frac{1}{2 R^{2} m_{\star}} \sim A^{-1 / 3} / R$, so

\footnotetext{
${ }^{10}$ If the amplitude of the surface waves were not small then volume-preserving oscillations require $\alpha_{00}=$ $-\sum_{l \geq 2, m}\left|\alpha_{l m}\right|^{2}$, but this constraint can be ignored at leading order.
} 
for large $A$ will be of lower energy than the collective surface modes. However, as discussed in the next section, in the regimes of interest in this paper, the cross sections for exciting them are much lower. Additionally, the properties of these modes are model-dependent for example, pairing interactions between constituents could lead to superfluidity, as arises in forms of SM nuclear matter.

\subsection{Inelastic scattering form factors}

The presence of these low-lying modes means that even low-velocity scattering processes may have sufficient energy to be inelastic. In particular, scattering off the long-wavelength collective modes, since these involve all of the constituents, will be coherently enhanced as for elastic scattering, and have their own momentum-dependent form factors.

Generally, this form factor can be calculated from the overlap of initial and final state wavefunctions. As derived in appendix A, if the scattering occurs through a scalar contact interaction with the constituents of the DM state, then (returning to the notation of section 2.1) the form factor for inelastic scattering into a one-phonon surface mode of angular momentum number $l$ is, as given in equation (A.8),

$$
F(q)=\frac{3 A}{\sqrt{4 \pi}} i^{l}(2 l+1)^{1 / 2} \epsilon_{l} j_{l}(q R)
$$

(as compared to equation (2.2) for elastic scattering), where $\epsilon_{l}$ is the natural amplitude associated with oscillations in that mode. This result is correct to first order in $\epsilon_{l}$, and valid for $q R \lesssim 1 / \epsilon_{l}$ (beyond that, the wavefunction overlap can be computed numerically). Excitations of multiple phonons are associated with further factors of $\epsilon_{l}$. Comparing elastic to inelastic scattering, we see that the latter has form factors corresponding to higher spherical Bessel functions, and is suppressed by powers of the natural amplitude of the surface modes; as per equation (3.8), this is parametrically small for large $A$.

The cross sections for scattering off single-particle excitations add incoherently, giving $\sigma \propto A$. There may also be further suppression factors. The case of degenerate fermionic matter may be well-approximated by scattering off a Fermi gas of non-interacting quasiparticles - as discussed in appendix B, this is, for low momentum transfers, strongly suppressed by degeneracy factors. Generally, incoherent scattering should only be significant for momentum transfers comparable to the Fermi momentum (and energy transfers comparable to the Fermi energy), or for very large composite states where scattering from collective modes is highly suppressed (appendix B).

\subsection{Inelastic recoil spectra}

If collisions are inelastic - say, the DM state is excited from $m_{X}$ to $m_{X}+\delta$ - then the minimum velocity [37] for which we can obtain a given recoil energy is ${ }^{11}$

$$
v_{\min }=\frac{1}{\sqrt{2 m_{N} E_{R}}}\left(\frac{m_{N} E_{R}}{\mu_{X N}}+\delta\right) .
$$

\footnotetext{
${ }^{11}$ Equation (3.10) is accurate up to multiplicative correction of size at most $\sim \sqrt{\delta / M}$ - this always gives a good approximation, since $\delta<\frac{1}{2} \mu_{X N} v_{\max }^{2}$ is required for any excitations to occur, and galactic escape velocity is deeply non-relativistic.
} 
We have $d E_{R}=\frac{2 p p^{\prime}}{m_{N}} \frac{d \Omega^{*}}{4 \pi}$, where $p$ and $p^{\prime}$ are the initial and final momentum in the CoM frame. The final state phase space available is also proportional to $p^{\prime}$, so the differential rates for elastic and inelastic scattering are related by

$$
\frac{d \sigma_{i}}{d E_{R}}=\frac{d \sigma_{e}}{d E_{R}} \frac{\left|\mathcal{M}_{i}\right|^{2}}{\left|\mathcal{M}_{e}\right|^{2}}
$$

where the $\mathcal{M}$ are the matrix elements. Aside from differences in the momentum-dependence of the matrix elements, giving different form factors, the main qualitative difference from elastic scattering is that $v_{\text {min }}$ is no longer monotonically increasing with $E_{R}$, instead having a minimum at $E_{R}=\delta \mu_{X N} / m_{N}$. This is in contrast to elastic scattering, where the fact that that $v_{\min }$ is monotonically increasing had important consequences (see sections 2.5 and 2.6) for data analysis.

As per above, we generically expect large composite states to have low-energy excitations, which may be excited by scattering with SM nuclei. The energy recoil spectrum will then be a sum over the spectra for scattering into each of these states (including the ground state, which gives elastic scattering). Labelling the states by $\lambda$,

$$
\frac{d R}{d E_{R}}=\frac{n_{X}}{2 \mu_{X n}^{2}} \sigma_{X n} F_{N}(q)^{2} \sum_{\lambda} g\left(v_{\min , \lambda}\left(E_{R}\right)\right) \frac{\left|C_{\lambda}\right|^{2}}{\left|C_{n}\right|^{2}} F_{X, \lambda}(q)^{2},
$$

where $\frac{C_{\lambda}}{C_{n}}$ gives the ratio of matrix elements (cf. equation (2.7)), and the $F_{X, \lambda}$ have a common normalisation such that elastic scattering has $F(0)=1$.

Figure 5 shows an example of the energy recoil spectrum arising from a uniform-density composite state of the kind discussed in section 3.1, in the regime where elastic scattering, and inelastic scattering into the first few surface modes, are the dominant effects. Due to the relative suppression of inelastic modes, from both form factor effects and the small amplitude of surface oscillations, elastic scattering generally dominates in such scenarios.

Finally we comment that the above analysis assumed that the DM particles are dominantly in the ground state. If de-excitation times are long enough, there is a possibility that there may be a significant cosmological population of excited DM states, altering the inelastic scattering phenomenology with exothermic interactions being possible. We leave discussion of this highly model-dependent possibility for future work.

\section{Astrophysical capture}

In addition to potentially being visible in direct detection experiments, interactions between DM and SM matter may also lead to the capture of DM by astrophysical objects, the most interesting generally being various kinds of stars. Once enough DM has accumulated inside the star, then either self-interactions among the DM (annihilations, self-scattering, etc.), or more complicated DM-SM interactions, may in some models lead to observable alterations of stellar properties. For definiteness, we consider the case of asymmetric DM, in which DM may build up in the star without being destroyed through annihilations.

For spatially-extended DM states, the possibility of large cross sections for inelastic DM-DM interactions may give rise to modifications of the distribution of captured DM 


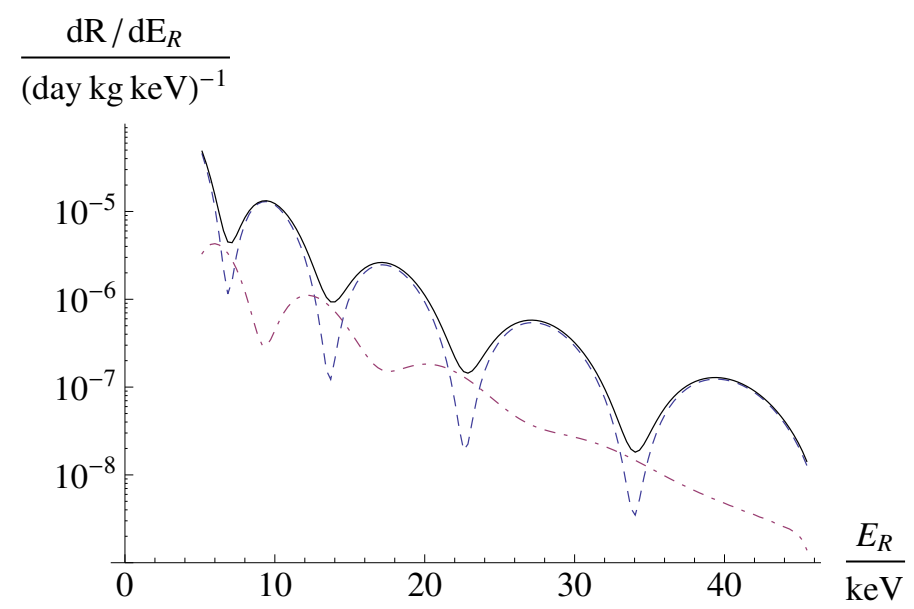

Figure 5. Energy recoil spectrum (Germanium target) for spherical DM state with parameters as per figure 2, incorporating elastic scattering and inelastic excitations of surface modes (black). Blue (dashed) and red (dot-dashed) curves show contributions from elastic and inelastic scattering respectively. The energy of the first surface mode is taken to be $28 \mathrm{keV}$, so a number of modes contribute at higher recoil energies, flattening out the inelastic recoil spectrum. The amplitude of the inelastic modes is set a factor $\sim 10$ above the hydrodynamic estimate from section 3.1, for illustrative purposes. Note that the form factor for the first excited mode is 90 degrees out of phase from that for elastic scattering - this effect can be seen in inelastic SM-SM scattering, e.g. figure 1 of [35].

inside the star, analogously to the possibility of effects on astrophysical halo shapes mentioned in [13]. In models with large velocity kicks after exothermic collisions, these could take the form of ejecting captured DM from the star, as discussed in e.g. [12]. Alternatively, other models could lead to the contraction of the captured DM distribution, potentially all of the way down to a very dense configuration at the centre of the star. Since we have been considering composite states composed of similar constituents, some kind of repulsive self-interaction countering the attractive binding forces is already required in such models, and will generally remove the danger of such a DM configuration collapsing into a black hole. ${ }^{12}$ In the absence of any further dynamics, there are no obvious externally observable consequences of a high-DM-density central region. In particular, the release of binding energy from fusions will have no significant effect on the star - for symmetric DM, almost all of the DM captured by the star can generally annihilate with no observable heating, and we are only injecting a small fraction of this energy. However, in the case of run-away fusions, there may be inelastic collisions between extremely large composite states, which could result in the release of large amounts of energy, in small volumes and on rapid timescales. If some of these de-excitation products couple to the SM strongly enough, the SM energy injection resulting from these processes may have consequences - for example, [40] points out that sufficiently fast and localised energy injection inside a white dwarf may ignite a Type 1a supernova, even in a sub-Chandrasekhar mass dwarf.

\footnotetext{
${ }^{12}$ As may occur in models of very heavy fundamental asymmetric DM [38, 39]
} 


\subsection{Effect of dark form factors on capture rate}

The composite nature of the DM may also affect the initial capture rate, through the momentum-dependence of scattering cross sections. From [41], the capture rate per unit volume is given by

$$
\frac{d C}{d V}=\int_{0}^{\infty} d u \frac{f(u)}{u} w \Omega(w),
$$

where $u$ is the velocity at infinity of the DM particle relative to the Sun, $f(u)$ is the DM speed distribution at infinity, $w$ is the velocity of the DM particle at the scattering location, and $\Omega(w)$ is the scattering rate to less than escape velocity off the material at that location. The scattering rate is

$$
\Omega(w)=\sum_{i} n_{i} w \Theta\left(E_{\max , i}-E_{\min }\right) \int_{E_{\min }}^{E_{\max , i}} d E \frac{d \sigma_{i}}{d E},
$$

where $n_{i}$ is the number density of SM nuclear species $i, E_{\min }=\frac{1}{2} m_{X} u^{2}$ is the minimum energy loss required for capture, and $E_{\max , i}=\frac{2 \mu_{i}^{2}}{m_{i}} w^{2}$ with $\mu_{i} \equiv m_{i} m_{X} /\left(m_{i}+m_{X}\right)$ is the maximum kinematically-allowed energy loss (taking the 'cold sun' approximation where the scattering nuclei are at rest). In the case where the scattering involves momentum dependent form factors, then using the notation of section 2.2 ,

$$
\Omega(w)=\sum_{i} n_{i} \frac{\sigma_{X i}}{2 \mu_{i}^{2} w} \Theta\left(q_{\max , i}-q_{\min , i}\right) \int_{q_{\min , i}}^{q_{\max , i}} q d q F_{i}(q)^{2} F_{X}(q)^{2},
$$

where $q_{\min , i}=\sqrt{m_{i} m_{X}} u$, and $q_{\max , i}=2 \mu_{i} w$.

Combining into a single form factor $F(q) \equiv F_{i}(q) F_{X}(q)$, if $F(q) \simeq 1$ for $q<1 / R$, then for $q_{\max , i} R=2 \mu_{i} w R \lesssim 1$ the form factor will have no significant effect. If $q_{\max , i} R \gg 1$, but $q_{\min , i} R \lesssim 1$, then assuming that $F(q)^{2}$ falls off faster than $1 / q^{2},{ }^{13}$ the dominant contribution to the integral will be from momenta $\sim 1 / R$, giving

$$
\int_{q_{\min , i}}^{q_{\max , i}} q d q F(q)^{2} \sim \frac{1}{R^{2}}
$$

If $q_{\min , i} R \gg 1$ as well, then the integral is

$$
\int_{q_{\min , i}}^{q_{\max , i}} q d q F(q)^{2} \sim q_{\min , i}^{2} F\left(q_{\min , i}\right)^{2} \Lambda\left(q_{\min , i}, q_{\max , i}\right)
$$

where $\Lambda$ will be $\sim 1$, if $q_{\min , i} \ll q_{\max , i}$ and $F(q)^{2}$ does not fall off significantly faster than a power law in $q$ (if $q_{\min , i} \geq q_{\max , i}$, then $\Lambda=0$ ). Turning to the integral over DM halo velocities, if the velocity distribution is steeply falling past some typical velocity $\bar{u}$ and approximately constant (in $d^{3} u$ space) below that, as for a Maxwell-Boltzmann distribution, then $f(u) \simeq n_{X} u^{2} / \bar{u}^{3}$, and we can approximate the integral as

$$
\frac{d C}{d V} \simeq \frac{n_{X}}{\bar{u}^{3}} \int_{0}^{\bar{u}} u d u w \Omega(w)
$$

\footnotetext{
${ }^{13}$ In particular, the Fourier transform of a spherically symmetric distribution with at worst step function discontinuities eventually falls off at least as fast as $1 / q^{2}$.
} 
If $q_{\max , i}(\bar{u}) R=2 \mu_{i} w(\bar{u}) R \lesssim 1$, then no part of this integral has any significant momentum dependence (for capture off species $i$ ). If $q_{\max , i} R \gg 1$ throughout (i.e. $2 \mu_{i} v_{\mathrm{esc}} R \gg 1$, where $v_{\text {esc }}$ is the escape velocity), but $q_{\min , i}(\bar{u}) R=\sqrt{m_{i} m_{X}} \bar{u} R \lesssim 1$, then the integral becomes

$$
\int_{0}^{\bar{u}} u d u w \Omega(w) \simeq n_{i} \frac{\sigma_{X i}}{2 \mu_{i}^{2}} \frac{1}{R^{2}} \frac{\bar{u}^{2}}{2} .
$$

For momentum-independent scattering with $q_{\max , i} \gg q_{\min , i}(\bar{u})$,

$$
\int_{0}^{\bar{u}} u d u w \Omega(w) \simeq n_{i} \frac{\sigma_{X i}}{2 \mu_{i}^{2}} 4 \mu_{i}^{2} v_{\mathrm{esc}}^{2} \frac{\bar{u}^{2}}{2}
$$

(where we assume that $v_{\text {esc }} \gg \bar{u}$, so that terms of the form $\bar{u}^{4}$ can be ignored), so the suppression relative to this case is $\frac{1}{4 \mu_{i}^{2} v_{\mathrm{esc}}^{2} R^{2}} \equiv \frac{1}{(k R)^{2}}$, where $k / 2=\mu_{i} v_{\mathrm{esc}}$ is approximately the initial momentum in the CoM frame.

If $q_{\min , i}(\bar{u}) R \gg 1$, then

$$
\begin{aligned}
\int_{0}^{\bar{u}} u d u w \Omega(w) \simeq & n_{i} \frac{\sigma_{X i}}{2 \mu_{i}^{2}}\left(\int_{0}^{1 /\left(\sqrt{m_{i} m_{X}} R\right)} u d u \frac{1}{R^{2}}+\right. \\
& \left.\int_{1 /\left(\sqrt{m_{i} m_{X}} R\right)}^{\bar{u}} u d u m_{i} m_{X} u^{2} F\left(\sqrt{m_{i} m_{X}} u\right)^{2} \Lambda\left(\sqrt{m_{i} m_{X}} u, 2 \mu_{i} w\right)\right) .
\end{aligned}
$$

If $F(q)^{2}$ drops off faster than $1 / q^{4}$, then this integral is dominated by $u \sim 1 /\left(\sqrt{m_{i} m_{X}} R\right)$, giving

$$
\sim n_{i} \frac{\sigma_{X i}}{2 \mu_{i}^{2}} \frac{1}{2 m_{i} m_{X} R^{4}}=n_{i} \frac{\sigma_{X i}}{2 \mu_{i}^{2}} \frac{1}{R^{2}} \frac{\bar{u}^{2}}{2}\left(\frac{1}{m_{i} m_{X} R^{2} \bar{u}^{2}}\right)
$$

which has an extra suppression factor. If, as in the Bessel-function case, $F(q)^{2} \propto 1 / q^{4}$ for large $q$, then the second integral in equation (4.9) contributes some logarithmic multiple of $1 /\left(m_{i} m_{X} R^{4}\right)$.

To summarise, for heavy DM $\left(m_{X} \gg m_{i}\right)$ with a radius of $R$ large enough to be probed in collisions, $k R \gg 1$, the elastic capture rate off species $i$ will be suppressed relative to momentum-independent scattering by $\sim(k R)^{-2} \min \left(1, \frac{\zeta}{m_{i} m_{X} R^{2} \bar{u}^{2}}\right)$, replacing the usual kinematic suppression ${ }^{14} \min \left(1, \frac{m_{i} v_{\mathrm{esc}}^{2}}{m_{X} \bar{u}^{2}}\right)$ (here, $\zeta$ is some logarithmic integral). As an example, if we suppose that the Sun (escape velocity 0.002c) has $k R \simeq 1$, then capture in white dwarfs $\left(v_{\text {esc }} \sim 0.02 c\right)$ is suppressed by a factor of $\sim 10^{-3}$, and in neutron stars $\left(v_{\text {esc }} \sim 0.7 c\right)$ by $\sim 10^{-5}$, compared to standard elastic scattering (for white dwarfs, the SM nuclear form factor is also important). These suppressions mean that inelastic scattering may be the dominant capture process, though we leave the investigation of this to future work.

\footnotetext{
${ }^{14}$ For momentum-independent scattering, if $q_{\min , i}(\bar{u})>q_{\max , i}$, then the integral in equation (4.8) is cut off above when $q_{\min , i}(u) \simeq q_{\max , i}$, i.e. when $u \sim \sqrt{m_{i} / m_{X}} v_{\text {esc }} \equiv u_{c}$ (taking $\left.\mu_{i} \simeq m_{i}\right)$. This reduces the value of the integral by a factor of $\sim u_{c}^{2} / \bar{u}^{2}=m_{i} v_{\mathrm{esc}}^{2} /\left(m_{X} \bar{u}^{2}\right)$.
} 


\subsection{Self-interactions of captured dark matter}

One possible effect of large DM self-interaction cross sections is to increase the capture rate, in that incoming DM can scatter off already-captured DM as well as off SM nuclei in the star. However, for heavy DM, and in locations of reasonable astrophysical DM density, it is generally hard to accumulate enough captured DM to have any significant effect on the overall capture rate. Supposing that the star has effective capture cross section $A_{s}$ for DM particles streaming through it, the total number of DM particles it captures in its lifetime is $N_{X} \sim A_{s} t_{s} v n_{X}$, where $n_{X}$ is the local DM number density and $v$ is the characteristic relative velocity (of order galactic orbital velocities, $\sim 220 \mathrm{~km} \mathrm{sec}^{-1}$ ). Writing the DM self-scattering cross-section as $\sigma_{X X}$, the total cross sectional area for DM-DM scattering is at most

$$
\begin{aligned}
A_{X} & =\sigma_{X X} N_{X} \sim\left(\sigma_{X X} / m_{X}\right) t_{s} v \rho_{X} A_{s} \\
& \simeq A_{s}\left(\frac{\sigma_{X X} / m_{X}}{\mathrm{barn} / \mathrm{GeV}}\right) \frac{v}{220 \mathrm{~km} \mathrm{sec}^{-1}} \frac{t_{s}}{5 \mathrm{Gyr}} \frac{\rho_{X}}{0.3 \mathrm{GeV} \mathrm{cm}^{-3}} .
\end{aligned}
$$

For elastically-scattering DM with velocity-independent self-scattering cross section, the observational limit on the self-scattering cross section is $\sigma_{X X} / m_{X} \lesssim 1 \mathrm{barn} / \mathrm{GeV}$ (see e.g. [42]). That $A_{X}$ can be of order $A_{s}$ for this value means that there are possible DM models in which self-capture is significant (e.g. [43, 44]). However, for large composite DM states, the fact that $\sigma_{X X} / m_{X} \propto m_{X}^{-1 / 3}$ for uniform matter means that large states generally stand little chance of having significant self-capture. Evaluating $\sigma_{X X} / m_{X}$ for a composite state of interior density $\rho_{b}$, and constituent number $A$,

$$
\frac{\sigma_{A A}}{m_{A}} \simeq \frac{0.05 \mathrm{barn}}{\mathrm{GeV}} A^{-1 / 3}\left(\frac{1 \mathrm{GeV}}{m_{1}}\right)^{1 / 3}\left(\frac{1 \mathrm{GeV} \mathrm{fm}^{-3}}{\rho_{b}}\right)^{2 / 3},
$$

we see that for SM-like or heavier constituents, large composite states are well below elastic self-interaction bounds, so do not give interesting self-capture effects.

However, interactions between already-captured DM states could potentially have interesting effects. Particularly interesting are interactions which may lead to 'run-away' effects, in which the DM distribution inside the star contracts to a very dense state. This could arise either from inelastic collisions which are dissipative (i.e. some of the initial KE is lost into de-excitation products), or from fusions, which result in heavier DM states that have a correspondingly smaller equilibrium radius inside the star. In sections 4.2.1 and 4.2.2, we will perform some approximate calculations to demonstrate the feasibility of these scenarios.

\subsubsection{Dissipative collisions}

For dissipative collisions, the dynamics are governed by the rate at which DM-DM collisions dissipate energy, versus the rate at which DM-SM collisions add it, re-thermalising the DM. As an estimate, if some fraction $\alpha$ of integrated phase space density for DM lies within a spatial radius $r$, then the rate of self-interactions is set by

$$
\Gamma_{X X} \sim \sigma_{X X} n_{X} v_{X} \sim \frac{\sigma_{X X}}{r^{2}} \alpha N_{X} \frac{3}{4 \pi} \sqrt{\frac{G M_{\star}}{R_{\star}^{3}}} \sim 10 \text { day }^{-1} \alpha \frac{A_{X}}{r^{2}},
$$


where we have taken $v_{X}^{2} \sim \frac{G M(r)}{r}$, and $A_{X}$ is as per equation (4.12). In thermal equilibrium with the SM matter in the star, the DM states would have an isothermal distribution,

$$
\rho_{X} \sim e^{-r^{2} /\left(2 r_{*}^{2}\right)}, \quad r_{*}=\left(\frac{3 T_{\star}}{4 \pi G m_{X} \rho_{\star}}\right)^{1 / 2},
$$

where $T_{\star}$ and $\rho_{\star}$ are the temperature and density of the stellar core. Evaluating $\Gamma_{X X}$ for $r=r_{*}$, and putting in values appropriate to the Sun,

$$
\Gamma_{X X} \sim 6 \mathrm{yr}^{-1} \alpha\left(\frac{\sigma_{X n} / m_{X}}{10^{-8} \mathrm{pb} / \mathrm{TeV}}\right)\left(\frac{m_{X}}{\mathrm{TeV}}\right)^{2 / 3}\left(\frac{1 \mathrm{GeV} \mathrm{fm}^{-3}}{\rho_{b}}\right)^{2 / 3}
$$

where we have used equation (D.1) for the solar capture rate, and we have replaced $\sigma_{X X}$ by the geometrical cross section between two spheres of mass $m_{X}$ and internal density $\rho_{b} .{ }^{15}$ This interaction timescale is much less than the lifetime of the Sun. So, as long as scattering with SM particles does not counteract the increase in the local DM number density that comes about from losing energy in inelastic scatterings, it is inconsistent for a large fraction of the captured DM to have a basically isotropic steady state phase space distribution with most of the density within a sphere of isothermal or smaller radius. Thus, if the DM-SM cross section is large enough, the DM distribution may contract down to a very dense configuration at the centre of the star.

It remains to check that SM scatterings do not re-thermalise DM fast enough to avoid this contraction. The viscous drag force on a DM state is approximately $\mathbf{f}_{\text {drag }} \sim$ $-m_{N} n_{N} v_{N} \sigma_{X N} \mathbf{v}_{X} \equiv-\gamma m_{X} \mathbf{v}_{X}$, where $m_{N}, n_{N}$ and $v_{N}$ are the mass, number density and average speed of the SM scatterers, $\sigma_{X N}$ is the momentum transfer cross section between $X$ and the scatterers, and $\mathbf{v}_{X}$ is the velocity of $X$ in the thermal rest frame of the scatterers. Thus, $\gamma$ gives the damping rate at which scatterings return $\mathbf{v}_{X}$ to the thermal distribution. For parameters relevant to the Solar core,

$$
\gamma \simeq \frac{m_{N} n_{N} v_{N} \sigma_{X N}}{m_{X}} \simeq 3 \times 10^{-4} \mathrm{yr}^{-1}\left(\frac{\sigma_{X n} / m_{X}}{10^{-8} \mathrm{pb} / \mathrm{TeV}}\right)
$$

Thus, as long as each inelastic collision does not dissipate too small a fraction of the initial KE $\left(\sim 10^{-4}\right.$ for the parameters in equations. (4.16) and (4.17)), SM-DM thermalisation will be too slow to prevent contraction. Also, since the thermalisation timescale is much less than the lifetime of the star, we do expect to contract down to isothermal densities in the first place (in particular, this means that we will lose almost all of any net angular momentum the captured DM distribution might have had initially, meaning that rotational support of the collapsing distribution will not be a worry). The same separation between self-interaction rates and thermalisation times may, for suitable parameter ranges, apply in the case of white dwarfs and neutron stars as well (however, for neutron stars there is an issue of whether thermalisation timescales are less than the lifetime of the star [45]).

\footnotetext{
${ }^{15} \mathrm{~A} 1 \mathrm{TeV}$ mass sphere of this density has radius $\simeq 6 \mathrm{fm}$, which is of the order of the inverse momentum transfer in the initial DM-nucleus collisions (for solar capture), so there will not be significant momentum suppression of the capture rate.
} 


\subsubsection{Fusions}

An alternative way to realise run-away contraction is for the DM states to progressively increase in mass through fusions, and correspondingly for their isothermal radius within the star to decrease. In contrast to the previous section, this scenario relies on thermalisation through SM-DM scattering being fast enough. It also relies on fusion cross sections being large enough, and on fusions remaining energetically favourable up to very large sizes - these features may naturally be realised in models of composite DM such as those discussed in [13].

As discussed in appendix $\mathrm{B}$, we expect the coherent momentum transfer cross section between DM and SM states to scale as $\sigma_{A N} \propto A^{2 / 3}$ for states $A$ with $p R_{A} \gg 1$, where $p$ is the characteristic momentum of the SM scatterers. Thus, $\gamma_{A} \simeq\left(A_{p} / A\right)^{1 / 3} \gamma_{A_{p}}$ for $A \gg A_{p}$, where $A_{p}$ is the size for which the DM states are of radius $\sim 1 / p$, i.e. $p R_{A_{p}} \sim 1$, with $\gamma$ given by equation (4.17). In terms of the size $A_{i}$ of states which have radius $\sim 1 / p_{i}$, where $p_{i}$ is the characteristic momentum of SM scatterers in the initial collision (which occurs at around the escape velocity), thermalisation is naively effective (assuming small enough injections of kinetic energy on the DM side) for $A \lesssim 10^{26} A_{i}\left(\frac{\sigma_{A_{i} n} / m_{A_{i}}}{10^{-8} \mathrm{pb} / \mathrm{TeV}}\right)^{3} \equiv A_{\mathrm{th}}$. The isothermal distribution for such heavy states may already have volume comparable to their saturated volume. If not, then further fusions will generally be fast enough to combine most of the DM into a few very large composites - the initial number density, and so rate of fusions, will be very high, with build-up continuing as $\Gamma_{A A} \propto A^{-1} A^{2 / 3} \sim A^{-1 / 3}$ from number density and cross section factors respectively.

For white dwarfs and neutron stars, we have a similar conclusion that fusions are potentially fast enough to form a very dense configuration. In these cases, the star is compact enough that even a DM distribution of radius comparable to the entire star can still have a high enough self-interaction rate for fusions to combine the majority of the DM into a dense state (though we would still need to worry about dissipating energy and angular momentum, especially in the neutron star case).

We note that the estimates in section 4.2.1 and 4.2.2 should be viewed as rough plausibility estimates - proper investigation of these issues would require realistic modelling of the DM-DM collisions, and of the phase space distribution of DM at each stage of the process. In particular, this section has ignored the possibility that DM collision types other than fusions are important (e.g. fragmentations etc.), and also assumed that the velocity kicks imparted by fusion de-excitation products are small enough to be re-thermalised quickly. However, the point was merely to illustrate that run-away contraction is a plausible possibility.

\section{Conclusions}

In this paper, we have investigated some of the consequences that follow if a proportion of DM is composed of large composite states - specifically, states consisting of a large number of constituents forming an extended, semi-uniform object with a saturated density.

The spatial extension of these objects introduces a dark form factor into elastic scattering amplitudes as discussed in section 2. This has two effects: first, coherently enhanced 
scattering rates with the result that collider bounds on DM cross sections are effectively weakened by factors of up to $1 / A$ (section 2.3). Second, the introduction of an additional characteristic momentum dependence, whose effects on direct detection energy recoil spectra, for DM states with radii large compared to SM nuclei, are considered in sections 2.3 and 2.4. We find that, in the most visible cases, such signals may be distinguishable from elastic scattering, in a halo-velocity-independent manner, with only $\mathcal{O}(50)$ scattering events (section 2.6).

Large composite states will generically have long-wavelength collective excitations, which give rise to the possibility of low-energy, coherently enhanced inelastic scattering processes, as discussed in section 3 . Though such processes will generally be sub-dominant in direct detection experiments (section 3.3), in specific models they may have signatures, in direct detection or astrophysically.

We considered the capture of DM by stars in section 4. The presence of dark form factors and inelastic scattering may alter the capture rate. In addition we investigated the possibility of inelastic interactions between captured DM states having an effect on the DM distribution inside the star. Strikingly, for plausible types of self-interactions, we argue that it is possible for almost all of the captured DM to accumulate into a very dense configuration (sections 4.2.1 and 4.2.2).

Finally, we emphasise that our calculations have focused on what we believe to be the leading, most model-independent features associated with the interaction of large composite DM states with the SM sector. In specific realisations of large composite DM there may well be other even more striking signals. It would clearly be interesting to construct specific models which realise large composite DM and investigate the resulting phenomenology.

\section{Acknowledgments}

We wish to thank Asimina Arvanitaki, Mads Frandsen, Peter Graham, Felix Kahlhoefer, Surjeet Rajendran and Ian Shoemaker for useful discussions, and Joan Elias Miro for useful comments on a draft. We are particularly grateful to John Cherry for detailed discussions of exposure times. RL and JMR thank the Stanford Institute for Theoretical Physics and the Perimeter Institute for Theoretical Physics, and SMW thanks the Oxford Physics Department, for hospitality during completion of this work. RL acknowledges support from an STFC studentship.

\section{A Matrix elements for inelastic scattering}

Suppose that we have a scattering process in which a state $i$, with initial momentum $\mathbf{k}$, scatters off a state $I$ with initial momentum $\mathbf{p}$ (all non-relativistic), resulting in final states $f$ and $F$ with momenta $\mathbf{k}^{\prime}$ and $\mathbf{p}^{\prime}$ respectively. The matrix element for this, in the Born approximation, is

$$
\begin{aligned}
\mathcal{A} & =\left\langle f, \mathbf{k}^{\prime} ; F, \mathbf{p}^{\prime}\left|H_{\mathrm{int}}\right| i, \mathbf{k} ; I, \mathbf{p}\right\rangle \\
& =\left\langle f, \mathbf{k} ; F, \mathbf{p}\left|e^{i \mathbf{q} \cdot\left(\hat{\mathbf{x}}_{F}-\hat{\mathbf{x}}_{r}\right)} H_{\mathrm{int}}\right| i, \mathbf{k} ; I, \mathbf{p}\right\rangle \\
& \equiv \int d \mathbf{r} e^{i \mathbf{q} \cdot \mathbf{r}} V_{\mathrm{eff}}(\mathbf{r}, \mathbf{v}),
\end{aligned}
$$


where $\mathbf{k}^{\prime}-\mathbf{k}=\mathbf{p}-\mathbf{p}^{\prime} \equiv \mathbf{q}$ is the momentum transfer, $\mathbf{v}$ is the relative velocity of $i$ and $I$, and $\left\langle\mathbf{x}_{f} ; \mathbf{x}_{F}\left|H_{\text {int }}\right| \mathbf{k} ; \mathbf{p}\right\rangle \equiv(2 \pi)^{-3} V_{\text {eff }}\left(\mathbf{x}_{f}-\mathbf{x}_{F}, \mathbf{v}\right) e^{i \mathbf{k} \cdot \mathbf{x}_{f}} e^{i \mathbf{p} \cdot \mathbf{x}_{F}}$. That is, the matrix element is given, as a function of the momentum transfer $\mathbf{q}$, by the Fourier transform of a (possibly velocity-dependent) effective potential $V_{\text {eff }}$, whose form will depend on the interaction Hamiltonian. If the initial or final states have directional properties (polarisations), $V_{\text {eff }}$ may also depend on those.

As discussed in section 3.1, the surface modes of an incompressible liquid drop can be obtained by quantising the classical surface oscillations, in which the surface is displaced as in equation (3.4). Classically, for a scalar interaction between a plane-wave scatterer and the nuclear matter, the Fourier transform of the interaction potential is given by

$$
\begin{aligned}
F\left(\alpha_{l m}\right) & =\int_{r<R^{\prime}(\theta, \phi)} d^{3} \mathbf{r} e^{i \mathbf{q} \cdot \mathbf{r}} V_{0} \\
& =V_{0} \int d \Omega \int_{0}^{R^{\prime}(\theta, \phi)} r^{2} d r \sum_{l^{\prime}}\left(2 l^{\prime}+1\right) i^{l^{\prime}} j_{l^{\prime}}(q r) P_{l^{\prime}}(\cos \theta),
\end{aligned}
$$

where we have taken $\mathbf{q}$ to be in the $\mathbf{z}$ direction. For $m \neq 0$, this integral is clearly zero. Expanding to first order in a given $\alpha_{l 0}$, and eliding $V_{0}$,

$$
\begin{aligned}
F\left(\alpha_{l 0}\right) & =F(0)+\sqrt{4 \pi} \int d \Omega \alpha_{l 0} R^{3} Y_{l 0}(\theta) \sum_{l^{\prime}}\left(2 l^{\prime}+1\right)^{1 / 2} i^{l^{\prime}} j_{l^{\prime}}(q R) Y_{l^{\prime} 0}(\theta) \\
& =F(0)+\alpha_{l 0} \frac{3 A}{\sqrt{4 \pi}}(2 l+1)^{1 / 2} i^{l} j_{l}(q R) .
\end{aligned}
$$

Treating each mode as a harmonic oscillator, we have $\hat{\alpha}_{l 0}=\epsilon_{l}\left(\hat{a}_{l}+\hat{a}_{l}^{\dagger}\right)$, where $\epsilon_{l}$ is the dimensionless amplitude from equation (3.7), and $\hat{a}_{l}^{\dagger}$ is the creation operator for the $l, 0$ mode. So the matrix element between the ground state and the first excited state is, to first order in $\epsilon_{l}$, given by

$$
\left\langle 1_{l}|F(\hat{\alpha})| 0\right\rangle=\frac{3 A}{\sqrt{4 \pi}}(2 l+1)^{1 / 2} i^{l} \epsilon_{l} j_{l}(q R) .
$$

Higher phonon number states are associated with further factors of $\epsilon_{l}$ (for the appropriate $l$ numbers), and more factors of $q R$ in front of the spherical Bessel function. For the expansion to make sense, we must have that $j_{l}(q r)$ varies slowly over the interval $R(1 \pm \epsilon)$, so we need $q R \lesssim \pi / \epsilon_{l}$. Beyond this approximation,

$$
\left\langle 1_{l}|F(\hat{\alpha})| 0\right\rangle=\int d \alpha \psi_{1}^{*}(\alpha) \psi_{0}(\alpha) F(\alpha),
$$

where the $\psi_{n}$ are the oscillator wavefunctions (for a harmonic oscillator, Hermite polynomials multiplied by an exponential), and $F(\alpha)$ is from equation (A.5) (note that the oscillator properties derived in section 3.1 are also to first order in $\left.\epsilon_{l}\right)$. In particular, comparing equation (A.8) to the elastic scattering form factor $3 A j_{1}(q R) /(q R)$ from equation (2.2), both are $\sim A \epsilon_{l}^{2}$ at $q R \sim 1 / \epsilon_{l}$, but the correct form of the inelastic form factor from equation (A.9) drops off faster than the elastic form factor beyond this, since the wavefunction for the surface displacement integrates over multiple, cancelling, Bessel-function periods. 


\section{B High-momentum scattering from large composite states}

In this appendix, we consider the scattering behaviour of large composite states when the momentum $p$ of the incoming scatterer is such that $p R \gg 1$, where $R$ is the radius of the composite state.

\section{B.1 Coherent scattering}

The elastic scattering form factor should fall off at least as fast as $(q R)^{-2}$. For thermalisation-type processes, we are most interested in the rate at which SM scatterings exchange energy-momentum with the composite state, which for dominantly soft scattering will be set parametrically by the momentum transfer cross section $\sigma_{\mathrm{tr}}=\int(1-\cos \theta) \frac{d \sigma}{d \Omega} d \Omega$. Writing $\frac{d \sigma}{d \Omega}=\frac{\sigma_{0}}{4 \pi} f(q R) /(q R)^{4}$, and using $q^{2}=2 \mu^{2} v^{2}(1-\cos \theta)$ in the CoM frame,

$$
\sigma_{\mathrm{tr}}=\frac{1}{2} \sigma_{0} \int d \cos \theta(1-\cos \theta) \frac{f(q R)}{(q R)^{4}}=\frac{1}{2} \frac{\sigma_{0}}{(\mu v R)^{4}} \int_{0}^{2 \mu v} \frac{d q}{q} f(q R) .
$$

The dimensionless integral on the r.h.s. provides a logarithmic factor $\Lambda$, giving

$$
\sigma_{\mathrm{tr}} \sim \frac{\Lambda}{(\mu v R)^{4}} \sigma_{0}=\frac{\Lambda}{(k R)^{4}} \sigma_{0} .
$$

Since $\sigma_{0} \propto A^{2}$, we have $\sigma_{\mathrm{tr}} \sim A^{2 / 3}$ for $A$ large enough that $k R \gg 1$ (with $\Lambda$ changing only logarithmically with $k R$ ), as used in section 4.2.2.

The above assumed that the form factor was that appropriate for plane-wave scattering. If both the composite state and the SM scatterer are better-localised than the size of the composite state, then the wavefunction overlap in the form factor will not probe the full composite state, giving different results.

For inelastic scattering, taking the composite state to be much heavier and much larger than the scattering state, we can approximate the scattering as being against an infinite uniform medium (assuming that $p$ is small enough that it does not resolve structure on the scale of individual constituents). Since the collective modes are linearly dispersing, $\omega=c_{c} k$, then by the usual pseudo-momentum and energy conservation considerations, scatterers can only excite these if their velocity relative to the medium is greater than $c_{c}$ (as per superfluids). Putting in some illustrative numbers, the speed of CNO nuclei inside the Sun is $v \simeq 3 \times 10^{-4} c$, while the speed of sound for compressional modes of SM-like nuclear matter is $\sim 0.1 c$.

For a composite state of finite radius, this corresponds to the fact that the modes of small enough energy to be excited have $k \ll q$, so the wavefunction overlap in the form factor is very small (the $q$ wave oscillates much faster than the $k$ wave, giving a large cancellation). With collective surface modes, as explained in appendix $\mathrm{A}$, for $\epsilon_{l} q R \gg 1$ there is again a large cancellation.

The above comments assume that the composite state is in its ground state. If there is some non-zero occupation number for high-wavenumber modes, then down-scatterings of these are energetically permitted, and will not suffer from the cancellation suppression described. We do not consider the case of scattering against excited states here, assuming that the de-excitation times are much shorter than the times between composite-SM collisions (for this to be the case, de-excitation will generally have to be to hidden sector states). 


\section{B.2 Incoherent scattering}

As discussed in section 3.1, as well as the collective modes, there may also be low-lying modes corresponding to the excitation of 'single constituents' - in the case of degenerate fermionic matter, excitations in which particles just below the Fermi surface are scattered to just above it. By the usual Fermi-liquid theory, ignoring interactions and approximating the scattering as occurring from a non-interacting Fermi gas should provide a good first approximation. [45] discusses scattering from a degenerate Fermi gas, finding that the scattering rate for low-energy scatterers is independent of the Fermi momentum (as can be seen from geometrical considerations). This can result in large suppression factors compared to the naive scattering rate given by $\sigma n v$ - for scatterers with momentum $k \ll$ $p_{F}$, and also low velocity compared to the Fermi velocity, we have an effective suppression (for energy transfer rates) of $\sim\left(\frac{k}{p_{F}}\right)^{4} \frac{m_{n}^{3}}{\mu^{2} m_{X}}$, where $m_{X}$ is the mass of the scatterer, $m_{n}$ is the effective mass of the quasi-particle, and $\mu=m_{X} m_{n} /\left(m_{X}+m_{n}\right)$ is the reduced mass.

Since the cross sections for scattering off single-particle excitations also add incoherently, $\sigma \propto A$, coherent scattering should dominate unless the size of the state is very large, so that as reviewed in the previous sub-section, the coherent transfer cross section grows slower than $A$. Making an estimate for SM-like nuclear matter (individual constituents of mass $m_{1}=1 \mathrm{GeV}$, bulk nuclear matter density $\rho=1 \mathrm{GeV} / \mathrm{fm}^{3}$ ) scattering off solar material, we would need, very roughly, $A \gtrsim 10^{32}$ for incoherent scattering to start dominating the transfer cross section (corresponding to $R \gtrsim 40 \mu \mathrm{m}$ ). This is well above the size beyond which thermalisations were found to be ineffective in section 4.2.2. However, it does illustrate why, for example, scattering of DM in neutron stars is dominated by incoherent scattering.

\section{Statistical identification of rising distributions}

Suppose that we have some (one-dimensional) data points, which we assume are IID samples from some probability distribution. We wish to test the hypothesis that this distribution is non-increasing, with respect to some appropriate function of the parameter.

If we expect plausible alternative distributions to feature only one prominent rising segment (for example, the recoil spectra considered in section 2 have successive Bessel function peaks suppressed by both the natural fall-off of the Bessel function, and the velocity distribution), a sensible approach is to locate the interval with the 'worst' bias towards its right-hand end, and ask what the probability is that a given sample from a candidate non-increasing distribution would have an interval 'that extreme'. ${ }^{16}$

A simple measure of how biased an interval is towards its right-hand end is simply the average position of the points within it. The non-increasing distribution maximising the probability of right-biased points is clearly the uniform distribution on the interval. If observations are binned, we can find the exact distribution for the average of bin mid-point

\footnotetext{
${ }^{16}$ This would be a poor approach if we expected the rises in the distribution to be e.g. a small periodic signal super-imposed on some larger background, or more generally any small but structured deviation from a larger background.
} 
positions, for some number $n$ of samples, by performing the convolution of the (binned) uniform distribution on the interval with itself $n$ times. In particular, if the bins are of uniform widths, this distribution can be computed analytically. If observations are not binned, then the distribution for the average of the positions is the (rescaled) Irwin-Hall distribution for $n$ points. In both the discrete and continuous cases, the null distribution is approximately normal for large $n$, with variance $1 / \sqrt{12 n}$ (on the interval $[0,1]$ ).

So, if we have $n$ samples with a mean position of $x$ (rescaling the interval to be of width 1), we can use the appropriate distribution to find the probability of a mean position $\geq x$ arising from a uniform distribution on that interval. The test statistic for the whole sample is then the minimum such $p$-value for each sub-interval within the sample (since it is clear that the worst sub-interval will always terminate either at the left sample end, or at the location of a point, there are a finite number of sub-intervals to test). To determine the distribution of this test statistic under a candidate non-increasing distribution, Monte Carlo simulation can be used. A uniform distribution is clearly the non-increasing distribution most likely to produce fake rises, and while the data may indicate that the underlying distribution is far from uniform, for reasonably small numbers of samples the $p$-values obtained by adopting a uniform null distribution are only slightly worse than those obtained by allowing a free null distribution constrained by the fit to data. In particular, we adopt a uniform null distribution for the calculations in figure 4 , and find that the $p$-values are generally no more than a factor $\sim 2$ worse.

One issue is that, in many physical cases, there will be some resolution associated with our points. Then, the distribution our samples are drawn from is some (positive) underlying distribution convolved by a (positive) detector response function, so must be smooth on scales of order the resolution. This means that rightwards bunching on such scales must be spurious, so should not be considered in our test. The simplest way to solve this issue, if we expect rising features in plausible alternative distributions to be on scales larger than the resolution, is to bin the points on around the resolution scale.

Quantitatively, the variance for the average of $n$ uniform random variables on $[0,1]$ is $\frac{1}{\sqrt{12 n}}$, and the expected average position from a linearly rising distribution is $\frac{1}{2}+\frac{1}{6}$, so we would expect to exclude the uniform distribution at a significance of $\sim 0.6 \sqrt{n}$ sigma. In the example from figure 4, with 100 events overall, we expect around 26 in the first rise, so we would expect to obtain around a $3 \sigma$ exclusion, assuming that the worst intervals arising from the null distribution are of approximately that many events. This is indeed what we observe, with the $p$-value CDF in the 100-sample case following very closely this normal approximation (the yellow curve in figure 4, lying almost entirely underneath the yellow points). The CDFs for the 30 and 50 sample cases are not so well approximated, since the 'worst interval' is more variable with small numbers of events.

\section{Solar capture of heavy WIMPS}

Here, we give a very brief review of the capture of weakly-interacting, massive dark matter particles by the Sun. For $X$ particles much heavier than any of the relevant SM nuclei within the Sun (here, this means $m_{X} \gtrsim 400 \mathrm{GeV}$ ), the rate of capture by the Sun scales as 
$C_{\odot} \sim \sigma_{X n} \rho_{X} m_{X}^{-2}$, where $\sigma_{X n}$ is the scattering cross section with SM nucleons (assumed to be elastic and spin-independent) $[41,46] .{ }^{17}$ Summing over capture rates from the various elements in the Sun (the main contributions are from the CNO elements) using a standard solar model [47], we obtain

$$
C_{\odot} \simeq 3 \times 10^{19} \sec ^{-1}\left(\frac{\sigma_{X n}}{10^{-8} \mathrm{pb}}\right)\left(\frac{1 \mathrm{TeV}}{m_{X}}\right)^{2},
$$

(taking the couplings to protons and neutrons to be the same). We have assumed that the DM velocity distribution follows the Standard Halo Model — since, for high-mass WIMPs, only the low-velocity part of the distribution can be captured by scattering events, modifications that affect the low-velocity distribution will alter the capture rate. direct detection experiments imply that $\sigma_{X n} \lesssim 10^{-8} \mathrm{pb} \frac{m_{X}}{1 \mathrm{TeV}}$ [48], and the age of the Sun is $t_{\odot} \simeq 5 \mathrm{Gyr}$, so the number of $X$ particles captured is $\lesssim 5 \times 10^{36}\left(\frac{1 \mathrm{TeV}}{m_{X}}\right)$. As a fraction of the total flux of $X$ particles hitting the Sun, we capture $\sim 3 \times 10^{-7}\left(\frac{\sigma_{X n} / m_{X}}{10^{-8} \mathrm{pb} / \mathrm{TeV}}\right)$.

Here, 'captured' means that the $X$ particles are in gravitationally bound orbits passing through the Sun. Subsequent scatterings with material in the Sun will reduce the size of these orbits further, and eventually the $X$ particles will (ignoring other interactions) settle into an isothermal distribution $\rho_{X} \sim e^{-r /\left(2 r_{*}^{2}\right)}$, with [49]

$$
r_{\star}=\left(\frac{3 T_{\odot}}{4 \pi G m_{X} \rho_{\odot}}\right)^{1 / 2} \simeq 2 \times 10^{-3} R_{\odot}\left(\frac{\mathrm{TeV}}{m_{X}}\right)^{1 / 2}\left(\frac{T_{\odot}}{10^{7} \mathrm{~K}}\right)^{1 / 2}\left(\frac{150 \mathrm{~g} \mathrm{~cm}^{-3}}{\rho_{\odot}}\right)^{1 / 2},
$$

(where the temperature and density are appropriate to the solar core). If $m_{X} \ll 100 \mathrm{TeV}$, then the initial orbits will be small enough that planetary perturbations can be mostly neglected, and then thermalisation occurs in less than the lifetime of the Sun if $\sigma_{X n} \gtrsim$ $3 \times 10^{-13} \mathrm{pb}\left(m_{X} / \mathrm{TeV}\right)^{3 / 2}$ [50]. For larger $m_{X}$, most of the initial orbits are large enough that the effect of Jupiter perturbs them so that they no longer pass through the Sun, resulting in most of them never thermalising [50].

Finally we remark that the above calculations apply to the case of capture by elastic scattering with SM nuclei. If there are sufficiently low-lying excitations that inelastic scatterings are possible, in either or both direct detection experiments and solar capture, this may change the possible parameter space. (See $[51,52]$ for related investigations of solar capture of inelastic DM.)

\footnotetext{
${ }^{17}$ One factor of $m_{X}^{-1}$ comes from the number density $n_{X}=\rho_{X} m_{X}^{-1}$, while another arises from the fact that heavier particles lose a smaller fraction of their kinetic energy in collisions with SM nuclei, so only the low-speed part of the WIMP velocity distribution can be captured. This also assumes that $\sigma_{X n}$ is low enough that the Sun is optically thin to $X$ particles, which is the case for $\sigma_{X n} \lesssim 10^{-3} \mathrm{pb}$.
} 
Open Access. This article is distributed under the terms of the Creative Commons Attribution License (CC-BY 4.0), which permits any use, distribution and reproduction in any medium, provided the original author(s) and source are credited.

\section{References}

[1] M. Pospelov and A. Ritz, Astrophysical Signatures of Secluded Dark Matter, Phys. Lett. B 671 (2009) 391 [arXiv:0810.1502] [INSPIRE].

[2] J.D. March-Russell and S.M. West, WIMPonium and Boost Factors for Indirect Dark Matter Detection, Phys. Lett. B 676 (2009) 133 [arXiv:0812.0559] [inSPIRE].

[3] W. Shepherd, T.M.P. Tait and G. Zaharijas, Bound states of weakly interacting dark matter, Phys. Rev. D 79 (2009) 055022 [arXiv: 0901.2125] [INSPIRE].

[4] H. Goldberg and L.J. Hall, A New Candidate for Dark Matter, Phys. Lett. B 174 (1986) 151 [INSPIRE].

[5] J.L. Feng, M. Kaplinghat, H. Tu and H.-B. Yu, Hidden Charged Dark Matter, JCAP 07 (2009) 004 [arXiv: 0905.3039] [INSPIRE].

[6] D.E. Kaplan, G.Z. Krnjaic, K.R. Rehermann and C.M. Wells, Atomic Dark Matter, JCAP 05 (2010) 021 [arXiv:0909.0753] [INSPIRE].

[7] M. Yu. Khlopov, Composite dark matter from stable charged constituents, arXiv:0806.3581 [INSPIRE].

[8] R. Laha and E. Braaten, Direct detection of dark matter in universal bound states, Phys. Rev. D 89 (2014) 103510 [arXiv:1311.6386] [INSPIRE].

[9] J.A. Frieman, G.B. Gelmini, M. Gleiser and E.W. Kolb, Solitogenesis: Primordial Origin of Nontopological Solitons, Phys. Rev. Lett. 60 (1988) 2101 [INSPIRE].

[10] A. Kusenko and M.E. Shaposhnikov, Supersymmetric Q balls as dark matter, Phys. Lett. B 418 (1998) 46 [hep-ph/9709492] [inSPIRE].

[11] G. Krnjaic and K. Sigurdson, Big Bang Darkleosynthesis, arXiv:1406.1171 [INSPIRE].

[12] W. Detmold, M. McCullough and A. Pochinsky, Dark Nuclei I: Cosmology and Indirect Detection, Phys. Rev. D 90 (2014) 115013 [arXiv:1406.2276] [InSPIRE].

[13] E. Hardy, R. Lasenby, J. March-Russell and S.M. West, Big Bang Synthesis of Nuclear Dark Matter, JHEP 06 (2015) 011 [arXiv:1411.3739] [INSPIRE].

[14] G. Gelmini, A. Kusenko and S. Nussinov, Experimental identification of nonpointlike dark matter candidates, Phys. Rev. Lett. 89 (2002) 101302 [hep-ph/0203179] [INSPIRE].

[15] S. Chang, A. Pierce and N. Weiner, Momentum Dependent Dark Matter Scattering, JCAP 01 (2010) 006 [arXiv:0908.3192] [inSPIRE].

[16] B. Feldstein, A.L. Fitzpatrick and E. Katz, Form Factor Dark Matter, JCAP 01 (2010) 020 [arXiv: 0908.2991] [INSPIRE].

[17] S.D. McDermott, H.-B. Yu and K.M. Zurek, The Dark Matter Inverse Problem: Extracting Particle Physics from Scattering Events, Phys. Rev. D 85 (2012) 123507 [arXiv:1110.4281] [INSPIRE].

[18] J.F. Cherry, M.T. Frandsen and I.M. Shoemaker, Halo Independent Direct Detection of Momentum-Dependent Dark Matter, JCAP 10 (2014) 022 [arXiv:1405.1420] [INSPIRE]. 
[19] A.L. Fitzpatrick, W. Haxton, E. Katz, N. Lubbers and Y. Xu, The Effective Field Theory of Dark Matter Direct Detection, JCAP 02 (2013) 004 [arXiv:1203.3542] [INSPIRE].

[20] G. Duda, A. Kemper and P. Gondolo, Model Independent Form Factors for Spin Independent Neutralino-Nucleon Scattering from Elastic Electron Scattering Data, JCAP 04 (2007) 012 [hep-ph/0608035] [INSPIRE].

[21] C. McCabe, The Astrophysical Uncertainties Of Dark Matter Direct Detection Experiments, Phys. Rev. D 82 (2010) 023530 [arXiv: 1005.0579] [INSPIRE].

[22] A. Djouadi, O. Lebedev, Y. Mambrini and J. Quevillon, Implications of LHC searches for Higgs-portal dark matter, Phys. Lett. B 709 (2012) 65 [arXiv:1112.3299] [INSPIRE].

[23] M. Kuhlen et al., Dark Matter Direct Detection with Non-Maxwellian Velocity Structure, JCAP 02 (2010) 030 [arXiv:0912.2358] [INSPIRE].

[24] J.I. Read, L. Mayer, A.M. Brooks, F. Governato and G. Lake, A dark matter disc in three cosmological simulations of Milky Way mass galaxies, Mon. Not. Roy. Astron. Soc. 397 (2009) 44 [arXiv: 0902. 0009] [INSPIRE].

[25] J. Diemand et al., Clumps and streams in the local dark matter distribution, Nature 454 (2008) 735 [arXiv:0805.1244] [INSPIRE].

[26] P.J. Fox, G.D. Kribs and T.M.P. Tait, Interpreting Dark Matter Direct Detection Independently of the Local Velocity and Density Distribution, Phys. Rev. D 83 (2011) 034007 [arXiv: 1011.1910] [INSPIRE].

[27] P.J. Fox, J. Liu and N. Weiner, Integrating Out Astrophysical Uncertainties, Phys. Rev. D 83 (2011) 103514 [arXiv:1011.1915] [InSPIRE].

[28] M.T. Frandsen, F. Kahlhoefer, C. McCabe, S. Sarkar and K. Schmidt-Hoberg, Resolving astrophysical uncertainties in dark matter direct detection, JCAP 01 (2012) 024 [arXiv:1111.0292] [INSPIRE].

[29] N. Bozorgnia, J. Herrero-Garcia, T. Schwetz and J. Zupan, Halo-independent methods for inelastic dark matter scattering, JCAP 07 (2013) 049 [arXiv: 1305. 3575] [INSPIRE].

[30] E. Del Nobile, G. Gelmini, P. Gondolo and J.-H. Huh, Generalized Halo Independent Comparison of Direct Dark Matter Detection Data, JCAP 10 (2013) 048 [arXiv:1306.5273] [INSPIRE].

[31] S. Scopel and K. Yoon, A systematic halo-independent analysis of direct detection data within the framework of Inelastic Dark Matter, JCAP 08 (2014) 060 [arXiv: 1405. 0364] [INSPIRE].

[32] J.F. Cherry et al., in preparation.

[33] A. Bohr and B. Mottelson, Nuclear Structure: Volume 2, Nuclear deformations, Benjamin, (1969).

[34] S.R. Coleman, Q Balls, Nucl. Phys. B 262 (1985) 263 [Erratum ibid. B 269 (1986) 744] [INSPIRE].

[35] L.J. Tassie, Scattering of Alpha-Particles by a Vibrational Nucleus, Aust. J. Phys. 15 (1962) 135.

[36] C. Mahaux, P.F. Bortignon, R.A. Broglia and C.H. Dasso, Dynamics of the shell model, Phys. Rept. 120 (1985) 1 [INSPIRE].

[37] D. Tucker-Smith and N. Weiner, Inelastic dark matter, Phys. Rev. D 64 (2001) 043502 [hep-ph/0101138] [INSPIRE]. 
[38] K.M. Zurek, Asymmetric Dark Matter: Theories, Signatures and Constraints, Phys. Rept. 537 (2014) 91 [arXiv: 1308.0338] [INSPIRE].

[39] C. Kouvaris, Limits on Self-Interacting Dark Matter, Phys. Rev. Lett. 108 (2012) 191301 [arXiv:1111.4364] [INSPIRE].

[40] P.W. Graham, S. Rajendran and J. Varela, Dark Matter Triggers of Supernovae, arXiv: 1505.0444

[41] A. Gould, Resonant Enhancements in WIMP Capture by the Earth, Astrophys. J. 321 (1987) 571 [INSPIRE].

[42] S. Tulin, H.-B. Yu and K.M. Zurek, Beyond Collisionless Dark Matter: Particle Physics Dynamics for Dark Matter Halo Structure, Phys. Rev. D 87 (2013) 115007 [arXiv: 1302.3898] [INSPIRE].

[43] A.R. Zentner, High-Energy Neutrinos From Dark Matter Particle Self-Capture Within the Sun, Phys. Rev. D 80 (2009) 063501 [arXiv:0907.3448] [InSPIRE].

[44] M. Taoso, F. Iocco, G. Meynet, G. Bertone and P. Eggenberger, Effect of low mass dark matter particles on the Sun, Phys. Rev. D 82 (2010) 083509 [arXiv: 1005.5711] [InSPIRE].

[45] B. Bertoni, A.E. Nelson and S. Reddy, Dark Matter Thermalization in Neutron Stars, Phys. Rev. D 88 (2013) 123505 [arXiv:1309.1721] [INSPIRE].

[46] A. Gould, Cosmological density of WIMPs from solar and terrestrial annihilations, Astrophys. J. 388 (1992) 338 [INSPIRE].

[47] J.N. Bahcall, A.M. Serenelli and S. Basu, New solar opacities, abundances, helioseismology and neutrino fluxes, Astrophys. J. 621 (2005) L85 [astro-ph/0412440] [INSPIRE].

[48] LUX collaboration, D.S. Akerib et al., First results from the LUX dark matter experiment at the Sanford Underground Research Facility, Phys. Rev. Lett. 112 (2014) 091303 [arXiv: 1310.8214] [INSPIRE].

[49] K. Griest and D. Seckel, Cosmic Asymmetry, Neutrinos and the Sun, Nucl. Phys. B 283 (1987) 681 [Erratum ibid. B 296 (1988) 1034] [INSPIRE].

[50] A.H.G. Peter, Dark matter in the solar system II: WIMP annihilation rates in the Sun, Phys. Rev. D 79 (2009) 103532 [arXiv:0902.1347] [INSPIRE].

[51] S. Nussinov, L.-T. Wang and I. Yavin, Capture of Inelastic Dark Matter in the Sun, JCAP 08 (2009) 037 [arXiv: 0905.1333] [inSPIRE].

[52] A. Menon, R. Morris, A. Pierce and N. Weiner, Capture and Indirect Detection of Inelastic Dark Matter, Phys. Rev. D 82 (2010) 015011 [arXiv:0905.1847] [INSPIRE]. 